\title{
Eulerian Gaussian beams for high-frequency wave propagation
}

\author{
Shingyu Leung ${ }^{1}$, Jianliang Qian², and Robert Burridge ${ }^{3}$
}

\begin{abstract}
We design an Eulerian Gaussian beam summation method for solving Helmholtz equations in the high-frequency regime. The traditional Gaussian beam summation method is based on Lagrangian ray tracing and local ray-centered coordinates. We propose a new Eulerian formulation of Gaussian beam theory which adopts global Cartesian coordinates, level sets, and Liouville equations, yielding uniformly distributed Eulerian traveltimes and amplitudes in phase space simultaneously for multiple sources. The time harmonic wavefield can be constructed by summing up Gaussian beams with ingredients provided by the new Eulerian formulation. The conventional Gaussian beam summation method can be derived from the proposed method. There are three advantages of the new method: (1) We have uniform resolution of ray distribution. (2) We can obtain wavefields excited at different sources by varying only source locations in the summation formula. (3) We can obtain wavefields excited at different frequencies by varying only frequencies in the summation formula. Numerical experiments indicate that the Gaussian beam summation method yields accurate asymptotic wavefields even at caustics. The new method may be used for seismic modeling and migration.
\end{abstract}

\section{INTRODUCTION}

The method of Gaussian beam summation is powerful for seismic wave modeling and migration in the high frequency regime; see Cerveny et al. (1982), Norris et al. (1987), White et al. (1987), Hill (1990), Alkhalifah (1995), Hill (2001), Gray (2005), and references therein. In contrast to the geometrical ray theory in which the ray amplitude is unbounded at caustics, a Gaussian beam constructed around a central ray always has guaranteed regular behavior at caustics, and interference of multiple arrivals is achieved by summing up a bundle of Gaussian beams. We propose a purely Eulerian Gaussian beam summation method that combines the Gaussian beam ansatz introduced in Ralston (1983) with the paraxial Liouville formulation developed recently in Qian and Leung $(2004,2006)$ and Leung et al. (2004). The resulting Eulerian method is easy to implement and computationally efficient.

Gaussian beams are approximate asymptotically valid solutions to hyperbolic partial differential equations which are concentrated near a single ray through the domain. The existence of such solutions has been known to the applied mathematician since the 1960s, and these solutions have been used to obtain results on propagation of singularities in hyperbolic PDEs (Hormander, 1971; Ralston, 1983). On the other hand, the Gaussian beam migration operator can be viewed as the adjoint of the Gaussian beam modeling operator. As an alternative to Kirchhoff depth migration, Gaussian beam migration can take into account multiarrivals systematically, yielding accurate and efficient imaging methods in complex media (Hill, 1990, 2001; Hale, 1992); this methodology has undergone extensive development in recent years (Albertin et al., 2004; Gray, 2005; Han and Wu, 2005; Zacek, 2005; Protasov and Tcheverda, 2006).

Traditional Gaussian beams are constructed by using local ray coordinates. As a result, one has to compute the normal distance from every observation point to the central ray of every Gaussian beam (Cerveny et al., 1982; George et al., 1987), which is computationally cumbersome and expensive. To overcome this difficulty, George et al. (1987) and Hill (1990) propose using local geographic coordinates in the vicinity of an observation point, which only partially solves the problem.

To implement Gaussian beam summation in a global Cartesian coordinate, we adopt the ansatz proposed in Ralston (1983) and Tanushev et al. (2007) to construct Gaussian beams along central rays without resorting to local ray-centered coordinates. Mathematically, this ansatz constructs an approximate traveltime function with an imaginary part as a Taylor expansion around a central ray by using traveltime derivatives on the central ray; to some extent, the approx-

Manuscript received by the Editor November 28, 2006; revised manuscript received March 27, 2007; published online August $23,2007$.

${ }^{1}$ University of California, Department of Mathematics, Irvine, California. E-mail: syleung@ math.uci.edu.

${ }_{3}^{2}$ Wichita State University, Department of Mathematics and Statistics, Wichita, Kansas. E-mail: qian@math.wichita.edu.

${ }^{3}$ Albuquerque, New Mexico. E-mail: burridge@erl.mit.edu.

(C) 2007 Society of Exploration Geophysicists. All rights reserved. 
imate traveltime function in the traditional Gaussian beam summation can be obtained from the new approximate traveltime function by using a local ray-centered coordinate transformation. To have an Eulerian formulation capturing multiple arrivals and caustics, we adopt Liouville equations in a paraxial setting (Qian and Leung, 2004, 2006; Leung et al., 2004) to parameterize multiple sources and receivers; see these papers and the references therein for recent progress in Eulerian geometrical optics.

We begin with an outline of the Lagrangian Gaussian beam summation method. Then we give details of the Eulerian Gaussian beam summation method based on level sets. We briefly describe numerical procedures for implementing the Eulerian Gaussian beam summation method. Numerical experiments demonstrate the effectiveness of the new method. See the list of symbols used.

\section{MATHEMATICAL FRAMEWORK}

We shall consider a time-harmonic wave problem in $n$ dimensions with one distinguished direction, the $z$-direction. We set up Cartesian coordinates $z, x_{1}, \ldots, x_{\bar{n}}$, where $\bar{n}=n-1$. In the first instance, we consider the physical domain to be $\mathcal{R}^{n}$, but this will be truncated and discretized later to allow numerical computations.

Because of the special rôle of $z$, we shall consider the domain more naturally as $\mathcal{R} \times \mathcal{R}^{\bar{n}}$ and use the notation $\tilde{\boldsymbol{x}}=(z, \boldsymbol{x})$ $=\left(z, x_{1}, \ldots, x_{\bar{n}}\right)$. In our equations, we shall consider vectors to be column vectors, but in the text, we shall usually write vectors in transposed form as rows. Thus, we will, for instance, write $\tilde{\boldsymbol{x}}=(z, \boldsymbol{x})$ $=\left(z, x_{1}, \ldots, x_{\bar{n}}\right)$ but regard both $\tilde{\boldsymbol{x}}$ and $\boldsymbol{x}$ as columns in matrix calculations unless explicitly transposed.

\section{Eikonal equations}

We consider the Helmholtz wave equation for the scalar wavefield $U(z, x, y, \omega)$,

$$
\begin{aligned}
& \nabla^{2} U(z, \boldsymbol{x}, \omega)+\frac{\omega^{2}}{v^{2}(z, \boldsymbol{x})} U(z, \boldsymbol{x}, \omega) \\
& \quad=-\delta\left(z-z_{s}\right) \delta\left(x_{1}-x_{s, 1}\right) \ldots \delta\left(x_{\bar{n}}-x_{s, \bar{n}}\right) .
\end{aligned}
$$

in the physical domain $\mathcal{R}^{n}$, where $\bar{n}=1$ or 2 for practical applications, $\omega$ is frequency, $v(z, x)$ the wave speed at point $(z, x)$, and $\left(0, x_{s}\right)$ the coordinates of a source point. Here and in what follows, we have written the argument $z$ first because of its distinguished role.

We use the standard geometric optics large- $\omega$ ansatz for $U$, given by

$$
U=\left[A(\widetilde{\boldsymbol{x}})+O\left(\frac{1}{\omega}\right)\right] \exp [\mathrm{i} \omega \tau(\widetilde{\boldsymbol{x}})] .
$$

Substituting equation 2 into equation 1 and equating the terms of orders $\omega^{-2}$ and $\omega^{-1}$ zero away from the source, we obtain the eikonal and transport equations for traveltime $\tau$ and amplitude $A$ :

$$
\begin{gathered}
(\widetilde{\nabla} \tau)^{2}=\frac{1}{v^{2}}, \\
\widetilde{\nabla} \tau \cdot \widetilde{\nabla} A+\frac{1}{2} A \widetilde{\nabla}^{2} \tau=0,
\end{gathered}
$$

with corresponding initial conditions. Here, we have written $\widetilde{\nabla}$ for $\left(\partial / \partial z, \partial / \partial x_{1}, \ldots, \partial / \partial x_{\bar{n}}\right)$ reserving $\boldsymbol{\nabla}$ for the $\bar{n}$-dimensional gradient operator $\left(\partial / \partial x_{1}, \ldots, \partial / \partial x_{\bar{n}}\right)$.

In seismic applications, we assume that $z$ is vertically down, and we shall assume that the traveltime field satisfies

$$
\frac{\partial \tau}{\partial z}>0
$$

i.e., rays are subhorizontal. In this case, we may rewrite the eikonal equation 3 as an evolution equation in depth (Gray and May, 1994; Qian and Symes, 2002; Symes and Qian, 2003),

$$
\begin{gathered}
\frac{\partial \tau}{\partial z}-\sqrt{\frac{1}{v^{2}}-(\boldsymbol{\nabla} \tau)^{2}}=0, \\
\tau\left(0, x_{1}, \ldots, x_{\bar{n}}\right)=\tau_{0}\left(x_{1}, \ldots, x_{\bar{n}}\right), \quad \operatorname{Im} \tau_{0} \geq 0, \\
\left.\left.\frac{\partial \tau}{\partial z}\right|_{z=0}=p_{0,0}\left(x_{1}, \ldots, x_{\bar{n}}\right)\right), \\
\left.\left.\nabla \tau\right|_{z=0}=p_{0}\left(x_{1}, \ldots, x_{\bar{n}}\right)\right),
\end{gathered}
$$

where $\tau_{0}(\boldsymbol{x})$, and $\boldsymbol{p}_{0}(x, y)$ are given complex-valued smooth functions satisfying the compatibility conditions,

$$
\begin{gathered}
\boldsymbol{\nabla} \tau_{0}=\boldsymbol{p}_{0}(x, y), \\
p_{0,0}(x, y)-\sqrt{\frac{1}{v^{2}(0, x, y)}-\boldsymbol{p}_{0}^{2}(x, y)}=0 .
\end{gathered}
$$

At a point source, $\left(0, \boldsymbol{x}_{s}\right)$, we specify initial conditions,

$$
\tau_{s}=\tau_{0}\left(\boldsymbol{x}_{s}\right)=\tau\left(0, \boldsymbol{x}_{s}\right)=0,
$$

$$
\widetilde{\boldsymbol{p}}_{s}=\widetilde{\boldsymbol{p}}\left(\boldsymbol{x}_{s}, \boldsymbol{v}_{s}\right)=\frac{\widetilde{\boldsymbol{t}}_{s}}{v\left(0, \boldsymbol{x}_{s}\right)}=\frac{\widetilde{\boldsymbol{v}}_{s}}{\mu_{s} v\left(0, \boldsymbol{x}_{s}\right)} .
$$

where

$$
\begin{aligned}
& \widetilde{\boldsymbol{p}}_{s}=\left(p_{s, 0}, \boldsymbol{p}_{s}\right), \quad \widetilde{\boldsymbol{t}}_{s}=\left(t_{s, 0}, \boldsymbol{t}_{s}\right)=\frac{\widetilde{\boldsymbol{v}}_{s}}{\mu}, \\
& \widetilde{\boldsymbol{t}}_{s}^{2}=1, \quad \boldsymbol{v}_{s}=\frac{\boldsymbol{t}_{s}}{t_{s, 0}}, \\
& \widetilde{\boldsymbol{v}}_{s}=\left(1, \boldsymbol{v}_{s}\right), \quad \mu_{s}=\sqrt{1+\boldsymbol{v}_{s}^{2}} .
\end{aligned}
$$

Here, $\widetilde{\boldsymbol{t}}_{s}$ is a unit vector with $t_{s, 0}>0, t_{0}$ is the cosine of the angle that $\widetilde{\boldsymbol{p}}$ makes with the $z$-direction, and $\mu=1 / t_{0}$. Notice that we have written $\widetilde{\boldsymbol{t}}_{s}^{2}=1$ instead of $|\boldsymbol{t}|=1$ because we want to keep our expressions analytic: for complex $\widetilde{\boldsymbol{t}},|\overrightarrow{\boldsymbol{t}}|$ might signify $\sqrt{\widetilde{\boldsymbol{t}} \widetilde{\boldsymbol{t}}}$ where $\widetilde{\boldsymbol{t}}^{\dagger}$ is the Hermitian transpose. Please refer to the list of symbols for these and other symbols.

To apply Gaussian beam theory (Ralston, 1983; Tanushev et al., 2007), we let the axis, or central ray, of a beam be given by $\boldsymbol{x}=\boldsymbol{X}(z)$, the corresponding value of $\boldsymbol{p}=\boldsymbol{P}(z)$, the traveltime by $\tau=T(z)$, and we introduce the Hamiltonian

$$
H(z, \boldsymbol{X}, \boldsymbol{P})=-P_{0}=-\sqrt{\frac{1}{v^{2}[z, \boldsymbol{X}(x)]}-\boldsymbol{P}(z)^{2}},
$$

where $P_{0}(z)=\tau_{z}[z, X(z)]$, and $\boldsymbol{P}(z)=\boldsymbol{\nabla} \tau[z, X(z)]$. 
We shall need the ray tracing system,

$$
\begin{aligned}
\dot{\boldsymbol{X}} & =H_{\boldsymbol{P}}=\frac{\boldsymbol{P}}{\sqrt{\frac{1}{v^{2}}-\boldsymbol{P}^{2}}}=\frac{\boldsymbol{t}}{t_{0}}=\boldsymbol{v},\left.\quad \boldsymbol{X}\right|_{z=0}=\boldsymbol{x}_{s} ; \\
\dot{\boldsymbol{P}} & =-H_{\boldsymbol{X}}=\frac{-v_{\boldsymbol{X}}}{v^{3} \sqrt{\frac{1}{v^{2}}-\boldsymbol{P}^{2}}}=\frac{-\mu v_{\boldsymbol{X}}}{v^{2}}, \\
\left.\boldsymbol{P}\right|_{z=0} & =\boldsymbol{v}_{s} / \mu_{s} v(0, \boldsymbol{x}) ; \\
\dot{T} & =\frac{1}{v^{2} \sqrt{\frac{1}{v^{2}}-\boldsymbol{P}^{2}}}=\frac{\mu}{v},\left.\quad T\right|_{z=0}=\tau_{0}\left(\boldsymbol{x}_{s}\right) ;
\end{aligned}
$$

here the $\operatorname{dot}(\cdot)$ denotes the total derivative with respect to $z$. We also have used $H_{P}$, for instance, to denote the matrix of partial derivatives $\boldsymbol{\partial} H / \boldsymbol{\partial} P_{i}, i=1, \ldots, \bar{n}$, and $\boldsymbol{P}^{2}$ to stand for $P_{1}^{2}+\ldots+P_{\bar{n}}^{2}$, etc. The trajectories $\boldsymbol{X}(z)$ are the rays, and the full trajectories $[\boldsymbol{X}(z), \boldsymbol{P}(z)]$ are characteristic strips which for brevity we shall refer to as xp-rays, i.e., trajectories in the $2 \bar{n}$-dimensional space of $(\boldsymbol{x}, \boldsymbol{p})$ which is essentially phase space. Later, when we have eliminated $\boldsymbol{p}$ in favor of $\boldsymbol{v}$, we shall refer to the corresponding trajectories as $x \nu$-rays.

To emphasize the dependence on the initial conditions, we will write $\boldsymbol{X}=\boldsymbol{X}(z)=\boldsymbol{X}\left(z ; \boldsymbol{x}_{s}, \boldsymbol{v}_{s}\right), \boldsymbol{P}=\boldsymbol{P}(z)=\boldsymbol{P}\left(z ; \boldsymbol{x}_{s}, \boldsymbol{v}_{s}\right)$, and $T=T(z)$ $=T\left(z ; \boldsymbol{x}_{s}, \boldsymbol{v}_{s}\right)$.

For later convenience, we define the $\bar{n} \times \bar{n}$ projection matrices $\boldsymbol{Q}_{\perp}$ and $\boldsymbol{Q}_{\|}$:

$$
Q_{\perp}=I-\frac{\boldsymbol{v} \nu^{T}}{\boldsymbol{v}^{2}}, \quad Q_{\|}=\frac{\boldsymbol{v} \nu^{T}}{\boldsymbol{v}^{2}}
$$

These are real, symmetric, and satisfy $\boldsymbol{Q}_{\perp}^{2}=\boldsymbol{Q}_{\perp}, \boldsymbol{Q}_{\|}^{2}=\boldsymbol{Q}_{\|}$, and $\boldsymbol{Q}_{\perp} \boldsymbol{Q}_{\|}=0$. For our purposes, we note that the eigenvalues of the matrix $u \boldsymbol{Q}_{\perp}+v \boldsymbol{Q}_{\|}$are $u$ repeated $\bar{n}-1$ times and the simple eigenvalue $v$, with respective eigenspaces perpendicular and parallel to $\boldsymbol{v}$ and $\operatorname{det}\left\{u \boldsymbol{Q}_{\perp}+v \boldsymbol{Q}_{\|}\right\}=u^{\bar{n}-1} v$, because $\boldsymbol{Q}_{\perp}$ projects onto an $\bar{n}-1$ dimensional eigenspace, whereas $\boldsymbol{Q}_{\|}$projects onto a $1 \mathrm{D}$ eigenspace.

Each $\boldsymbol{x}_{s}, \boldsymbol{v}_{s}$ defines an axisymmetric Gaussian beam through $\boldsymbol{x}_{s}$ in the direction $\widetilde{\boldsymbol{t}}=(1, \boldsymbol{v}) / \mu$, waist centered at $\boldsymbol{x}_{s}$ and width determined by the positive parameter $\epsilon$. Associated with each such beam is a field of $x p$-rays, parameterized by an $\bar{n}$-vector $\boldsymbol{\alpha}=\left(\boldsymbol{\alpha}_{1}, \ldots, \boldsymbol{\alpha}_{\bar{n}}\right)$, and containing the central $x p$-ray, which has initial conditions $(\boldsymbol{X}(0), \boldsymbol{P}(0))=\left[\boldsymbol{x}_{s}, \boldsymbol{t}_{s} / v\left(0, \boldsymbol{x}_{s}\right)\right]$, but with neighboring members of the field having slightly different initial conditions $\boldsymbol{X}\left(0 ; \boldsymbol{x}_{s}, \boldsymbol{v}_{s}, \boldsymbol{\alpha}\right)=\boldsymbol{x}_{s}$ $+\left(\boldsymbol{Q}_{\perp}+\mu \boldsymbol{Q}_{\|}\right) \boldsymbol{\alpha}, \boldsymbol{P}\left(0 ; \boldsymbol{x}_{s}, \boldsymbol{t}_{s}, \boldsymbol{\alpha}\right)=\boldsymbol{t}_{s} / v\left(0, \boldsymbol{x}_{s}\right)+\mathrm{i} \epsilon\left(\boldsymbol{Q}_{\perp}+\mu^{-1} \boldsymbol{Q}_{\|}\right) \boldsymbol{\alpha}$. For each $\boldsymbol{x}_{s}, \boldsymbol{\nu}_{s}$ we shall be concerned with the variations of $\boldsymbol{X}\left(z ; \boldsymbol{x}_{s}, \boldsymbol{v}_{s}, \boldsymbol{\alpha}\right)$ and $\boldsymbol{P}\left(z ; \boldsymbol{x}_{s}, \boldsymbol{\nu}_{s}, \boldsymbol{\alpha}\right)$ along the ray with respect to $\boldsymbol{\alpha}$ at $\boldsymbol{\alpha}=0$, and we define $\boldsymbol{B}\left(z ; \boldsymbol{x}_{s}, \boldsymbol{v}_{s}\right)=\boldsymbol{\partial P}\left(z ; \boldsymbol{x}_{s}, \boldsymbol{v}_{s}, \boldsymbol{\alpha}\right) /\left.\boldsymbol{\partial} \alpha\right|_{\boldsymbol{\alpha}=0}$ and $\boldsymbol{C}\left(z ; \boldsymbol{x}_{s}, \boldsymbol{v}_{s}, \boldsymbol{\alpha}\right)$ $=\boldsymbol{\partial} \boldsymbol{X}\left(z ; \boldsymbol{x}_{s}, \boldsymbol{v}_{s}\right) /\left.\boldsymbol{\partial} \alpha\right|_{\alpha=0}$.

The dynamic ray tracing (DRT) system for these variations is obtained by differentiating equation 13 with respect to $\boldsymbol{\alpha}$ :

$$
\begin{aligned}
& \dot{\boldsymbol{B}}(z)=-H_{X \boldsymbol{P}} \boldsymbol{B}-H_{X X} \boldsymbol{C}, \quad \boldsymbol{B}(0)=i \epsilon\left(\boldsymbol{Q}_{\perp}+\frac{1}{\mu} \boldsymbol{Q}_{\|}\right), \\
& \dot{\boldsymbol{C}}(z)=H_{P \boldsymbol{P}} \boldsymbol{B}+H_{\boldsymbol{P} X} \boldsymbol{C}, \quad \boldsymbol{C}(0)=\boldsymbol{Q}_{\perp}+\mu \boldsymbol{Q}_{\|},
\end{aligned}
$$

where $\boldsymbol{\epsilon}>0$ and $\mu=\sqrt{1+\boldsymbol{v}^{2}}=1 / \cos \boldsymbol{\theta}, \boldsymbol{\theta}$ being the angle that the ray direction $\tilde{\boldsymbol{t}}$ makes with the $z$ direction. The initial values $\boldsymbol{B}(0)$,
$\boldsymbol{C}(0)$ ensure that the beam has circular symmetry about its axial direction $\widetilde{\boldsymbol{t}}$. In the DRT system equations 15 , the second derivatives of $H$ are

$$
\begin{aligned}
& H_{\boldsymbol{P P}}=\frac{v\left[\left(1-v^{2} \boldsymbol{P}^{2}\right) \boldsymbol{I}+v^{2} \boldsymbol{P} \boldsymbol{P}^{T}\right]}{\left(1-v^{2} \boldsymbol{P}^{2}\right)^{3 / 2}}, \\
& H_{\boldsymbol{X} \boldsymbol{X}}=\frac{\left(1-v^{2} \boldsymbol{P}^{2}\right)\left(v v_{\boldsymbol{X}}-3 v_{X} v_{X}^{T}\right)+v_{X} v_{X}^{T}}{v^{3}\left(1-v^{2} \boldsymbol{P}^{2}\right)^{3 / 2}}, \\
& H_{\boldsymbol{P} \boldsymbol{X}}=H_{\boldsymbol{X} \boldsymbol{P}}^{T}=\frac{\boldsymbol{P} v_{\boldsymbol{X}}^{T}}{\left(1-v^{2} \boldsymbol{P}^{2}\right)^{3 / 2}} .
\end{aligned}
$$

We notice for future reference that $\left.\boldsymbol{B} \boldsymbol{C}^{-1}\right|_{z=0}$ is i $\epsilon$ times the positive definite real symmetric matrix $\boldsymbol{Q}_{\perp}+\boldsymbol{Q}_{\|} / \mu^{2}$.

In the neighborhood of the source, the beam has width on the order of $1 / \sqrt{\omega \epsilon}$ and is confined to a circular cylindrical region with axis in the direction of $\tilde{\boldsymbol{t}}$. This cylinder cuts the $\bar{n}$-plane $z=0$ in an ellipsoidal region similar to the ellipsoid $\boldsymbol{x}^{T}\left(\boldsymbol{Q}_{\perp}+\boldsymbol{Q}_{\|} / \mu^{2}\right) \boldsymbol{x}=1$ with major and minor semi-axes proportional to $\mu$ parallel and 1 perpendicular to $\boldsymbol{v}$. It follows that this same cylindrical region cuts a right cross section, i.e., the section by the plane $\tilde{\boldsymbol{t}}^{T}\left(\widetilde{\boldsymbol{x}}-\widetilde{\boldsymbol{x}}_{s}\right)=0$ through the source, in an $\bar{n}$-sphere. We take all the beams to have axial symmetry and to be identical except for the directions $\widetilde{\boldsymbol{t}}_{s}$ of their axes. Each beam has a family of rays associated with it, and we shall parameterize this family of rays by the coordinates $\boldsymbol{\alpha}$ on the right cross section through the source.

The symplectic structure $\left[H_{P P}\right.$, and $-H_{X X}$ are symmetric, and $\left.-H_{X P}=-\left(H_{P X}\right)^{T}\right]$ of equations 15 implies, as we have just pointed out, that $\operatorname{Im}\left\{\boldsymbol{B}(z) \boldsymbol{C}(z)^{-1}\right\}$ remains symmetric and positive definite if, as implied by the DRT system equations 15 , it is symmetric and positive definite initially.

Because $\tau_{x}=\boldsymbol{p}, \tau_{x x}=\delta \boldsymbol{p} / \delta \boldsymbol{x}=(\boldsymbol{\partial} \boldsymbol{p} / \boldsymbol{\partial} \alpha)(\boldsymbol{\partial} \boldsymbol{x} / \boldsymbol{\partial} \alpha)^{-1}=\boldsymbol{B} \boldsymbol{C}^{-1}$, thus we have the following Taylor series approximation in the neighborhood of $\boldsymbol{x}=\boldsymbol{X}$,

$$
\begin{aligned}
\tau\left(z, \boldsymbol{x} ; \boldsymbol{x}_{s}, \boldsymbol{v}_{s}\right)= & T\left(z ; \boldsymbol{x}_{s}, \boldsymbol{v}_{s}\right)+\frac{1}{v \mu} \boldsymbol{N}^{T}(z)[\boldsymbol{x}-\boldsymbol{X}(z)] \\
& +\frac{1}{2}[\boldsymbol{x}-\boldsymbol{X}(z)]^{T} \boldsymbol{B}(z) \boldsymbol{C}^{-1}(z)[\boldsymbol{x}-\boldsymbol{X}(z)],
\end{aligned}
$$

where $\boldsymbol{X}, \boldsymbol{P}, \boldsymbol{B}$ and $\boldsymbol{C}$ also depend on $\left(z, \boldsymbol{x}_{s}, \boldsymbol{v}_{s}\right)$ but not on $\boldsymbol{\alpha}$, and we have expressed $\boldsymbol{P}$ in terms of $\boldsymbol{N}: \boldsymbol{P}=\boldsymbol{N} /(v \mu)$.

Let us express the direction of the central ray of a beam in terms of $\boldsymbol{v}$, and eliminate $\boldsymbol{p}$ in favor of $\boldsymbol{v}$ as follows in $v \widetilde{\boldsymbol{p}}=\widetilde{\boldsymbol{t}}=\left(t_{0}, \boldsymbol{t}\right)$ $=(1, \boldsymbol{v}) / \mu$, where $\boldsymbol{t}=\boldsymbol{v} \boldsymbol{p}=\boldsymbol{v} / \mu$. Then the ray tracing system 13 becomes

$$
\dot{X}=N, \quad \dot{N}=\frac{\mu^{2}}{v}\left(v_{z} N-v_{X}\right), \quad \dot{T}=\frac{\mu}{v},
$$

with initial conditions

$$
\boldsymbol{X}\left(0 ; \boldsymbol{x}_{s}, \boldsymbol{v}_{s}\right)=\boldsymbol{x}_{s}, \quad \boldsymbol{N}\left(0 ; \boldsymbol{x}_{s}, \boldsymbol{v}_{s}\right)=\boldsymbol{v}_{s}, \quad T\left(0 ; \boldsymbol{x}_{s}, \boldsymbol{v}_{s}\right)=0 .
$$

In the ray tracing system of equations 18 and the initial conditions equation 19, $\boldsymbol{X}$ and $\boldsymbol{N} \in \mathcal{R}^{\bar{n}}$ and the dependent variables are regarded as functions of $\left(z, \boldsymbol{x}_{s}, \boldsymbol{v}_{s}\right)$. We repeat the DRT system, equations 15: 


$$
\begin{aligned}
& \dot{\boldsymbol{B}}(z)=-H_{\boldsymbol{X} \boldsymbol{P}} \boldsymbol{B}-H_{X \boldsymbol{X}} \boldsymbol{C},\left.\quad \boldsymbol{B}(z)\right|_{z=0}=i \epsilon\left(\boldsymbol{Q}_{\perp}+\frac{1}{\mu} \boldsymbol{Q}_{\|}\right), \\
& \dot{\boldsymbol{C}}(z)=H_{\boldsymbol{P P}} \boldsymbol{B}+H_{\boldsymbol{P} X} \boldsymbol{C},\left.\quad \boldsymbol{C}(z)\right|_{z=0}=\left(\boldsymbol{Q}_{\perp}+\mu \boldsymbol{Q}_{\|}\right),
\end{aligned}
$$

where

$$
\begin{aligned}
& H_{P P}=v \mu\left(\boldsymbol{I}+N \boldsymbol{N}^{T}\right)=v \mu\left(\boldsymbol{Q}_{\perp}+\mu^{2} \boldsymbol{Q}_{\|}\right), \\
& H_{X P}=\frac{\mu^{2} v_{X} \boldsymbol{N}^{T}}{v}, \\
& H_{X X}=\frac{\mu\left(v v_{X X}-3 v_{X} v_{X}^{T}\right)+\mu^{3} v_{X} v_{X}^{T}}{v^{3}},
\end{aligned}
$$

and

$$
\begin{gathered}
v=v[z, X(z)], \quad \mu=\sqrt{1+N^{2}}, \quad Q_{\|}=\frac{N N^{T}}{N^{2}}, \\
Q_{\perp}=I-Q_{\|} .
\end{gathered}
$$

Finally, the amplitude along the axial ray is

$A\left(z ; \boldsymbol{x}_{s}, \boldsymbol{v}_{s}\right)=\sqrt{\frac{v(z, \boldsymbol{X}(z)) t_{0}\left(0 ; \boldsymbol{x}_{s}, \boldsymbol{v}_{s}\right) \operatorname{det}\left\{\boldsymbol{C}\left(0 ; \boldsymbol{x}_{s}, \boldsymbol{v}_{s}\right)\right\}}{v\left(0, \boldsymbol{x}_{s}\right) t_{0}\left(z ; \boldsymbol{x}_{s}, \boldsymbol{v}_{s}\right) \operatorname{det}\left\{\boldsymbol{C}\left(z ; \boldsymbol{x}_{s}, \boldsymbol{v}_{s}\right)\right\}}}$,

which is finite and nonzero everywhere (see Appendix A).

We recognize $t_{s, 0} \operatorname{det}\{\boldsymbol{C}(0)\}$ as the differential area of the right cross section of the beam at the source, which has been arranged to be the same for all beams. Thus, we may write

$A\left(z ; x_{s}, \boldsymbol{v}_{s}\right)=\sqrt{\frac{\text { const.v }(z, \boldsymbol{X}(z))}{v\left(0, \boldsymbol{x}_{s}\right) t_{0}\left(z ; \boldsymbol{x}_{s}, \boldsymbol{v}_{s}\right) \operatorname{det}\left\{\boldsymbol{C}\left(z ; \boldsymbol{x}_{s}, \boldsymbol{v}_{s}\right)\right\}}}$.

The previous derivation is based on the paraxial assumption, the so-called subhorizontal condition; see the inequality equation 4 . When the paraxial assumption does not apply, we may construct the Gaussian beam summation directly by using the approach presented in Ralston (1983) and Tanushev et al. (2007).

\section{Lagrangian Gaussian beam superposition}

The wavefield resulting from one Gaussian beam parameterized with initial takeoff direction $\widetilde{\boldsymbol{t}}_{\boldsymbol{s}}$ is

$$
\begin{aligned}
\Psi\left(z, \boldsymbol{x} ; \boldsymbol{x}_{s}, \boldsymbol{v}_{s}\right)= & \Psi_{0} \sqrt{\frac{v[z, \boldsymbol{X}(z)]}{v\left(0, \boldsymbol{x}_{s}\right) t_{0}\left(z ; \boldsymbol{x}_{s}, \boldsymbol{v}_{s}\right) \operatorname{det}\left\{\boldsymbol{C}\left(z ; \boldsymbol{x}_{s}, \boldsymbol{v}_{s}\right)\right\}}} \\
& \times \exp \left[i \omega \tau\left(z, \boldsymbol{x} ; \boldsymbol{x}_{s}, \boldsymbol{v}_{s}\right)\right]
\end{aligned}
$$

where $\boldsymbol{\tau}\left(z, \boldsymbol{x} ; \boldsymbol{x}_{s}, \boldsymbol{v}_{s}\right)$ is given by the Taylor series approximation (equation 17) with $\boldsymbol{P}(z)=N /(\mu v)$, and the radiation factor from equation B-18 is

$$
\Psi_{0}=\frac{\mathrm{i}}{4 \pi}\left(\frac{\omega}{2 \pi v_{s}}\right)^{n-2}
$$

and from equation B-20 we have

$$
\begin{aligned}
\Psi\left(z, \boldsymbol{x} ; \boldsymbol{x}_{s}, \boldsymbol{v}_{s}\right)= & \frac{-\mathrm{i}}{4 \pi}\left(\frac{\omega}{2 \pi v_{s}}\right)^{n-2} \sqrt{\frac{\mu(z) v[z, \boldsymbol{X}(z)]}{v\left(0, \boldsymbol{x}_{s}\right) \operatorname{det}\{\boldsymbol{C}\}}} \\
& \times \exp \left[\mathrm{i} \omega\left(\tau\left(z, \boldsymbol{x} ; \boldsymbol{x}_{s}, \boldsymbol{v}_{s}\right)\right] .\right.
\end{aligned}
$$

To compute the wavefield generated by a point source at $\boldsymbol{x}_{s}$, we integrate over beams with central rays emanating from the source in all the possible directions with $t_{0}$ positive. However, because of the rapid decay of amplitude in each beam away from the axis, only beams with axes passing close to $(z, x)$ contribute. From equations 17 and 27 we have

$$
\begin{aligned}
U\left(z, \boldsymbol{x} ; \boldsymbol{x}_{s}\right)= & \frac{\mathrm{i}}{4 \pi}\left(\frac{\omega}{2 \pi v_{s}}\right)^{n-2} \\
& \times \int_{-\infty}^{\infty} \cdots \int_{-\infty}^{\infty} \sqrt{\frac{\mu\left(z, \boldsymbol{x}_{s}, \boldsymbol{v}_{s}\right) v[z, \boldsymbol{X}(z)]}{v\left(0, \boldsymbol{x}_{s}\right) \operatorname{det}\left\{\boldsymbol{C}\left(z, \boldsymbol{x}_{s}, \boldsymbol{v}_{s}\right)\right\}}} \\
& \times \frac{\mathrm{d} \nu_{s, 1} \ldots \mathrm{d} \nu_{s, \bar{n}}}{\mu_{s}^{n}} \times \exp \left\{\mathrm { i } \omega \left(T\left(z ; \boldsymbol{x}_{s}, \boldsymbol{v}_{s}\right)\right.\right. \\
& +\frac{1}{v \mu} N^{T}\left(z, \boldsymbol{x}_{s}, \boldsymbol{v}_{s}\right)\left[\boldsymbol{x}-\boldsymbol{X}\left(z, \boldsymbol{x}_{s}, \boldsymbol{v}_{s}\right)\right] \\
& +\frac{1}{2}\left[\boldsymbol{x}-\boldsymbol{X}\left(z, \boldsymbol{x}_{s}, \boldsymbol{v}_{s}\right)\right]^{T} \boldsymbol{B}\left(z, \boldsymbol{x}_{s}, \boldsymbol{v}_{s}\right) \boldsymbol{C}^{-1}\left(z, \boldsymbol{x}_{s}, \boldsymbol{v}_{s}\right) \\
& \left.\times\left[\boldsymbol{x}-\boldsymbol{X}\left(z, \boldsymbol{x}_{s}, \boldsymbol{v}_{s}\right)\right]\right\} .
\end{aligned}
$$

Here, $\mathrm{d} \boldsymbol{v}_{s, 1} \ldots \mathrm{d} \boldsymbol{v}_{s, \bar{n}} / \mu_{s}^{n}$ is the solid angle element of integration on the $\bar{n}$-hemisphere $\left(1, \boldsymbol{v}_{s}\right) / \mu_{s}$ and $\mu_{s}=\sqrt{1+\boldsymbol{v}_{s}^{2}}=1 / \cos \boldsymbol{\theta}_{s}, \boldsymbol{\theta}_{s}$ being the angle the tangent to the ray $\widetilde{\boldsymbol{t}}$ makes with the $z$-axis at $\left(0, \boldsymbol{x}_{s}\right)$.

What does equation 28 mean? From uniqueness for system equations 18 , given $\boldsymbol{x}_{s}$ and $\boldsymbol{t}_{s}$ with $t_{s, 0}>0$, there is just one $x \boldsymbol{\nu}$-ray emanating from the source $\boldsymbol{x}_{s}$ with takeoff direction $\widetilde{\boldsymbol{t}}_{s}=\widetilde{\boldsymbol{v}}_{s} / \mu_{s}$. It pierces $z$ $=$ constant in the point $\left[z, \boldsymbol{X}\left(z ; \boldsymbol{x}_{s}, \boldsymbol{v}_{s}\right), \boldsymbol{N}\left(z ; \boldsymbol{x}_{s}, \boldsymbol{v}_{s}\right)\right]$. As $\boldsymbol{v}_{s}$ varies over $\mathcal{R}^{\bar{n}},\left[\boldsymbol{X}\left(z ; \boldsymbol{x}_{s}, \boldsymbol{v}_{s}\right), \boldsymbol{N}\left(z ; \boldsymbol{x}_{s}, \boldsymbol{v}_{s}\right)\right]$ traces out an $\bar{n}$-dimensional surface through

$$
\gamma\left(z ; \boldsymbol{x}_{s}\right)=\left\{\left[X\left(z ; \boldsymbol{x}_{s}, \boldsymbol{v}_{s}\right), N\left(z ; \boldsymbol{x}_{s}, \boldsymbol{v}_{s}\right)\right]: \boldsymbol{v}_{s} \in \mathcal{R}^{\bar{n}}\right\}
$$

in $\mathcal{R}^{2 \bar{n}}$, and $\gamma\left(0 ; \boldsymbol{x}_{s}\right)=\left\{\left(\boldsymbol{x}_{s}, \boldsymbol{v}_{s}\right): \boldsymbol{v}_{s} \in \mathcal{R}^{\bar{n}}\right\}$ is a vertical $\bar{n}$-plane in $\mathcal{R}^{2 \bar{n}}$. We note that $\gamma\left(z ; \boldsymbol{x}_{s}\right)$ can be considered as a surface parameterized by $\boldsymbol{v}_{s}$, whereas the $z$-variable indicates the evolution of $\gamma\left(0 ; \boldsymbol{x}_{s}\right)$ under the ray tracing equations 18 . Consequently, the summation formula (equation 28) states that at depth $z$ the integration is carried out with respect to the parameter $\boldsymbol{v}_{s}$ over the $\bar{n}$-surface $\gamma\left(z ; \boldsymbol{x}_{s}\right)$.

To carry out the above summation process, we need to choose a numerical quadrature formula, solve the ray tracing equations and the dynamical ray tracing system with takeoff directions at the quadrature sampling points, construct a Gaussian beam along each ray, and sum up the Gaussian beams to obtain the wavefield at each observation point. Because the above process is based on the Lagrangian ray tracing, it inherits some shortcomings from the approach, such as shadow zones and a nonuniform distribution of rays. Therefore, we look for an Eulerian approach to Gaussian beam superposition. 


\section{EULERIAN GAUSSIAN BEAM METHOD}

\section{Paraxial Liouville equations}

We summarize the level set based Eulerian method for computing multivalued traveltimes; see Qian and Leung $(2004,2006)$ and Leung et al. (2004) for details.

Let the vector fields $\boldsymbol{u}$ and $\boldsymbol{w}$ be defined from the ray tracing equations 18 as

$$
\boldsymbol{u}(z, \boldsymbol{x}, \boldsymbol{v})=\boldsymbol{v}, \quad \boldsymbol{w}=\frac{\mu^{2}}{v}\left[v_{z} \boldsymbol{v}-v_{X}\right] .
$$

Then the total derivative of any scalar or matrix function $\phi(z, \boldsymbol{x}, \boldsymbol{v})$ of $z, \boldsymbol{x}, \boldsymbol{v}$ along any $x \boldsymbol{\nu}$-ray trajectory is given by

$$
\frac{\mathrm{D} \phi}{\mathrm{D} z}=\phi_{z}+\boldsymbol{u}^{T} \phi_{x}+\boldsymbol{w}^{T} \phi_{\boldsymbol{v}}
$$

Let us assume that this rate of change is zero:

$$
\frac{\mathrm{D} \phi}{\mathrm{D} z}=\phi_{z}+\boldsymbol{u}^{T} \phi_{\boldsymbol{x}}+\boldsymbol{w}^{T} \phi_{\boldsymbol{v}}=0 .
$$

Then the value of $\phi$ at any point $(z, \boldsymbol{x}, \boldsymbol{v})$ is the same as its value at the initial point $\left(0, \boldsymbol{x}_{s}, \boldsymbol{v}_{s}\right)$ on the $x \boldsymbol{\nu}$-ray through $(z, \boldsymbol{x}, \boldsymbol{v})$. We may set $\phi(0, x, v)=f(x, v)$ to be any function of $(\boldsymbol{x}, \boldsymbol{v})$. Then $\phi(z, x, \boldsymbol{v})$ $=\boldsymbol{f}\left(\boldsymbol{x}_{s}, \boldsymbol{v}_{s}\right)$, where $\left(0, \boldsymbol{x}_{s}, \boldsymbol{v}_{s}\right)$ is the initial point on the unique $x \boldsymbol{\nu}$-ray through $(z, x, v)$. In particular, we shall later take $f(x, v)=x$ and $f(x, v)=v$.

On the other hand, suppose that the total $z$ derivative along a trajectory is not zero, but for instance $\mu / v(z, \boldsymbol{x})$ and the initial value is 0 , then the solution $\mathcal{T}(z, \boldsymbol{x}, \boldsymbol{v})$ of

$$
\frac{\mathrm{D} \mathcal{T}}{\mathrm{D} z}=\mathcal{T}_{z}+\boldsymbol{u}^{T} \mathcal{T}_{\boldsymbol{x}}+\boldsymbol{w}^{T} \mathcal{T}_{\boldsymbol{v}}=\frac{\mu}{v(z, \boldsymbol{x})}
$$

is equal to the traveltime $\boldsymbol{\tau}(z, \boldsymbol{x}, \boldsymbol{v})$ to $(z, \boldsymbol{x}, \boldsymbol{v})$ from the initial point (a source point) on the ray through $(z, \boldsymbol{x}, \boldsymbol{v})$ because its initial value 0 and total derivative along the ray are the same as for $\boldsymbol{\tau}$.

We may find the initial point on the ray by solving

$$
\frac{\mathrm{D} \mathcal{X}^{(0)}}{\mathrm{D} z}=\boldsymbol{\mathcal { X }}_{z}^{(0)}+\boldsymbol{u}^{T} \boldsymbol{\mathcal { X }}_{\boldsymbol{x}}^{(0)}+\boldsymbol{w}^{T} \boldsymbol{\mathcal { X }}_{\boldsymbol{v}}^{(0)}=\mathbf{0},
$$

for $\boldsymbol{\mathcal { X }}^{(0)}$ with initial value

$$
\mathcal{X}^{(0)}(0, \boldsymbol{x}, \boldsymbol{v})=\boldsymbol{x} .
$$

We may also find the direction parameter $\boldsymbol{\nu}_{s}$ on the ray by solving

$$
\frac{\mathrm{D} \mathcal{N}^{(0)}}{\mathrm{D} z}=\mathcal{N}_{z}^{(0)}+\boldsymbol{u}^{T} \mathcal{N}_{\boldsymbol{x}}^{(0)}+\boldsymbol{w}^{T} \mathcal{N}_{\boldsymbol{v}}^{(0)}=\mathbf{0}
$$

for $\mathcal{N}^{(0)}$ with initial value

$$
\mathcal{N}^{(0)}(0, \boldsymbol{x}, \boldsymbol{v})=\boldsymbol{v}
$$

Given $z>0, \boldsymbol{x}$ and $\boldsymbol{x}_{s}$, and having $\mathcal{T}(z, \boldsymbol{x}, \boldsymbol{\nu})$ and $\boldsymbol{\mathcal { X }}^{(0)}(z, \boldsymbol{x}, \boldsymbol{\nu})$, we may find the values $\boldsymbol{\nu}^{*}$ of $\boldsymbol{\nu}$ for which $\boldsymbol{\mathcal { X }}^{(0)}\left(z, \boldsymbol{x}, \boldsymbol{\nu}^{*}\right)=\boldsymbol{x}_{s}$, and there may be several. For each of these $\boldsymbol{\nu}^{*}$, we have the traveltime $\mathcal{T}\left(z, \boldsymbol{x}, \boldsymbol{\nu}^{*}\right)$ from point $\left(0, \boldsymbol{x}_{s}\right)$ to point $(z, \boldsymbol{x})$ with $z>0$. We have solved for multiple sources simultaneously and also dealt with the possibility of multiple arrivals at $(z, \boldsymbol{x})$ from $\left(0, \boldsymbol{x}_{s}\right)$. There will, of course, be computational restrictions relating to whether or not rays remain in the computational domain for all intermediate values of $z$.

Similarly, we may use equations of the form given by equation 33 to solve the dynamic ray tracing equations. Thus, we have the following equations for $\mathcal{B}(z, \boldsymbol{x}, \boldsymbol{\nu})$ and $\mathcal{C}(z, \boldsymbol{x}, \boldsymbol{\nu})$,

$$
\begin{gathered}
\mathcal{B}_{z}+\boldsymbol{u}^{T} \mathcal{B}_{\boldsymbol{x}}+\boldsymbol{w}^{T} \mathcal{B}_{\boldsymbol{v}}=-H_{x p} \mathcal{B}-H_{x \boldsymbol{x}} \boldsymbol{C}, \\
\mathcal{B}(0, \boldsymbol{x}, \boldsymbol{v})=i \epsilon\left(\boldsymbol{Q}_{\perp}+\frac{1}{\mu} \boldsymbol{Q}_{\|}\right) \\
\mathcal{C}_{z}+\boldsymbol{u}^{T} \mathcal{C}_{\boldsymbol{x}}+\boldsymbol{w}^{T} \mathcal{C}_{\boldsymbol{v}}=H_{\boldsymbol{p} \boldsymbol{p}} \mathcal{B}+H_{x p} \mathcal{C} \\
\mathcal{C}(0, \boldsymbol{x}, \boldsymbol{v})=\left(\boldsymbol{Q}_{\perp}+\mu \boldsymbol{Q}_{\|}\right)
\end{gathered}
$$

where $\epsilon>0$, and

$$
\begin{gathered}
H_{p p}=v \mu\left(\boldsymbol{Q}_{\perp}+\mu \boldsymbol{Q}_{\|}\right), \quad H_{x p}=\frac{\mu^{2} v_{x} \boldsymbol{v}^{T}}{v}, \\
H_{x x}=\frac{\mu\left(v v_{x x}-3 v_{x} v_{x}^{T}\right)+\mu^{3} v_{x} v_{x}^{T}}{v^{3}} .
\end{gathered}
$$

Let us now express equation 28 in terms of the quantities considered in this section; namely,

$$
\begin{aligned}
U\left(z, \boldsymbol{x}_{0} ; \boldsymbol{x}_{s}\right)= & \frac{\mathrm{i}}{4 \pi}\left(\frac{\omega}{2 \pi v_{s}}\right)^{n-2} \\
& \times \int_{\Gamma\left(z, \boldsymbol{x}_{s}\right)} \sqrt{\frac{\mu(z, \boldsymbol{x}, \boldsymbol{v}) v(z, \boldsymbol{x})}{v\left(0, \boldsymbol{x}_{s}\right) \operatorname{det}\{\mathcal{C}(z, \boldsymbol{x}, \boldsymbol{v})\}}} \\
& \times \frac{\mathrm{d} \mathcal{N}_{1}^{(0)} \ldots \mathrm{d} \mathcal{N}_{\bar{n}}^{(0)}}{\left(\mu^{(0)}\right)^{n}} \exp \{\mathrm{i} \omega[\mathcal{T}(z ; \boldsymbol{x}, \boldsymbol{v}) \\
& +\frac{1}{v \mu} \boldsymbol{v}^{T}\left(\boldsymbol{x}_{0}-\boldsymbol{x}\right)+\frac{1}{2}\left(\boldsymbol{x}_{0}-\boldsymbol{x}\right)^{T} \\
& \left.\left.\times \boldsymbol{B}(z, \boldsymbol{x}, \boldsymbol{v}) \mathcal{C}^{-1}(z, \boldsymbol{x}, \boldsymbol{v})\left(\boldsymbol{x}_{0}-\boldsymbol{x}\right)\right]\right\},
\end{aligned}
$$

where

$$
\mu=\sqrt{1+\boldsymbol{v}^{2}} \text { and } \mu^{(0)}=\sqrt{1+\left(\mathcal{N}^{(0)}\right)^{2}},
$$

and the domain $\Gamma\left(z, x_{s}\right)$ of integration is the $\bar{n}$-dimensional surface corresponding to $\gamma$ of equation 29 , or

$$
\Gamma\left(z, \boldsymbol{x}_{s}\right)=\left\{(\boldsymbol{x}, \boldsymbol{v}): \boldsymbol{\mathcal { X }}^{(0)}(z, \boldsymbol{x}, \boldsymbol{v})=\boldsymbol{x}_{s}\right\} .
$$

At this point, we leave the calculation for general dimension $n$ and specialize to $n=2$. Then $\widetilde{n}=1$ and $\mathbf{Q}_{\|}, B$ and $C$ reduce to scalars whereas $\mathbf{Q}_{\perp}$ does not exist.

\section{SPECIALIZATION TO 2D}

In two dimensions $\boldsymbol{x}, \boldsymbol{X}$ become single horizontal components $x, X$

$$
\begin{aligned}
& t_{0} \Rightarrow \cos \theta=1 / \mu, \\
& t \Rightarrow t_{1}=\sin \theta, \\
& \boldsymbol{\nu} \Rightarrow \nu=\frac{t_{1}}{t_{0}}=\tan \theta .
\end{aligned}
$$


The ray equations 18 and their initial conditions equations 19 become

$$
\begin{array}{r}
\dot{X}=\tan \theta, \quad \dot{N}=\frac{\mu^{2}}{v}\left(v_{z} N-v_{X}\right), \quad \dot{T}=\frac{1}{v \cos \theta}, \\
X\left(0 ; \boldsymbol{x}_{s}, \boldsymbol{v}_{s}\right)=x_{s}, \quad N\left(0 ; x_{s}, \nu_{s}\right)=\nu_{s}, \quad T\left(0 ; x_{s}, \nu_{s}\right)=0 .
\end{array}
$$

Because $N=\tan \theta$ and $\mu^{2}=\sec ^{2} \theta$, we can easily eliminate $N$ in favor of $\theta$; writing

$$
\begin{gathered}
\dot{X}=\tan \theta, \quad \dot{\theta}=\frac{1}{v}\left(v_{z} \tan \theta-v_{X}\right), \quad \dot{T}=\frac{1}{v \cos \theta}, \\
X\left(0 ; \boldsymbol{x}_{s}, \theta_{s}\right)=x_{s}, \quad \theta\left(0 ; x_{s}, \theta_{s}\right)=\theta_{s}, \quad T\left(0 ; x_{s}, \theta_{s}\right)=0,
\end{gathered}
$$

we may define

$$
u=\tan \theta, \quad w=\frac{1}{v}\left(v_{z} \tan \theta-v_{X}\right) .
$$

Then equations $34-37$ lead to

$$
\begin{aligned}
& \frac{\mathrm{D} \mathcal{X}^{(0)}}{\mathrm{D} z} \equiv \mathcal{X}_{z}^{(0)}+u^{T} \mathcal{X}_{x}^{(0)}+w^{T} \mathcal{X}_{\theta}^{(0)}=0, \quad \mathcal{X}^{(0)}(0, x, \theta)=x, \\
& \frac{\mathrm{D} \vartheta^{(0)}}{\mathrm{D} z} \equiv \vartheta_{z}^{(0)}+u^{T} \vartheta_{x}^{(0)}+w^{T} \vartheta_{\theta}^{(0)}=0, \quad \vartheta^{(0)}(0, x, \theta)=\theta,
\end{aligned}
$$

and equation 33 leads to

$$
\begin{gathered}
\frac{\mathrm{D} \mathcal{T}^{(0)}}{\mathrm{D} z} \equiv \mathcal{T}_{z}^{(0)}+u^{T} \mathcal{T}_{x}^{(0)}+w^{T} \mathcal{T}_{\theta}^{(0)}=\frac{\mu}{v(z, \boldsymbol{x})}, \\
\mathcal{T}^{(0)}(0, x, \theta)=0 .
\end{gathered}
$$

Before writing the dynamic ray tracing equations, note that when $n=2 \boldsymbol{Q}_{\perp}$ disappears and $\boldsymbol{Q}_{\|}=1$. Thus equations 39 become

$$
\begin{aligned}
H_{p p} & =\frac{v}{\cos ^{3} \theta}, \quad H_{x p}=\frac{v_{x} \tan \theta}{v \cos ^{2} \theta}, \quad H_{x x} \\
& =\frac{\cos ^{2} \theta\left(v v_{x x}-3 v_{x} v_{x}^{T}\right)+v_{x} v_{x}^{T}}{v^{3} \cos ^{3} \theta} .
\end{aligned}
$$

Equations 38 become

$$
\begin{aligned}
\mathcal{B}_{z}+u \mathcal{B}_{x}+w \mathcal{B}_{\theta} & =-H_{x p} \mathcal{B}-H_{x x} \mathcal{C}, \\
\mathcal{B}(0, x, \theta) & =\mathrm{i} \epsilon \cos \theta \\
\mathcal{C}_{z}+u \mathcal{C}_{x}+w \mathcal{C}_{\theta} & =H_{p p} \mathcal{B}+H_{x p} \mathcal{C}, \\
\mathcal{C}(0, \boldsymbol{x}, \theta) & =\frac{1}{\cos \theta}
\end{aligned}
$$

with $u, w, H_{x p}, H_{x x}, H_{p p}$ given in equations 50 and 52. Equation 40 now becomes

$$
\begin{aligned}
U\left(z, x_{0} ; \boldsymbol{x}_{s}\right)= & \frac{\mathrm{i}}{4 \pi} \int_{(x, \theta) \in \Gamma\left(z, x_{s}\right)} \sqrt{\frac{v(z, x)}{v\left(0, x_{s}\right) \mathcal{C}(z, x, \theta) \cos \theta}} \mathrm{d} \vartheta^{(0)} \\
& \times \exp \left\{\mathrm { i } \omega \left[\mathcal{T}(z ; x, \theta)+\frac{\sin \theta}{v}\left(x_{0}-x\right)\right.\right. \\
& \left.\left.+\frac{1}{2} \mathcal{B}(z, x, \theta) \mathcal{C}^{-1}(z, x, \theta)\left(x_{0}-x\right)^{2}\right]\right\}
\end{aligned}
$$

where the domain of integration is the curve $\Gamma\left(z, x_{s}\right)$ in the $2 \mathrm{D}$ $x \theta$-space:

$$
\Gamma\left(z, x_{s}\right)=\left\{(x, \theta): \mathcal{X}^{(0)}(z, x, \theta)=x_{s}\right\} .
$$

Because we may compute $\vartheta^{(0)}(z, x, \theta)$ from equations 50 , we may regard equation 54 as a Stieltjes integral. To compute it numerically, we would assume that discrete points $\left(x_{l}, \theta_{l}\right), l=1, \ldots, L$ are known on $\Gamma\left(z, x_{s}\right)$ and that they are sorted by increasing $\vartheta_{l}^{(0)}=\vartheta^{(0)}\left(z, x_{l}, \theta_{l}\right)$. Then the integral may be approximated by

$$
\begin{aligned}
& U\left(z, x_{0} ; \boldsymbol{x}_{s}\right)=\frac{\mathrm{i}}{8 \pi \sqrt{v\left(0, x_{s}\right)}} \\
& \times\left[\left(\vartheta_{2}^{(0)}-\vartheta_{1}^{(0)}\right) \sqrt{\frac{v\left(z, x_{1}\right)}{\left.\mathcal{C}\left(z, x_{1}, \theta_{1}\right) \cos \theta_{1}\right\}}}\right. \\
& \times \exp \left\{\mathrm { i } \omega \left[\mathcal{T}\left(z ; x_{1}, \theta_{1}\right)+\frac{\sin \theta_{1}}{v}\left(x_{0}-x_{1}\right)\right.\right. \\
& \left.\left.+\frac{1}{2} \mathcal{B}\left(z, x_{1}, \theta_{1}\right) \mathcal{C}^{-1}\left(z, x_{1}, \theta_{1}\right)\left(x_{0}-x_{1}\right)^{2}\right]\right\} \\
& +\sum_{l=2}^{L-1}\left(\vartheta_{l+1}^{(0)}-\vartheta_{l-1}^{(0)}\right) \sqrt{\frac{v\left(z, x_{l}\right)}{\left.\mathcal{C}\left(z, x_{l}, \theta_{l}\right) \cos \theta_{l}\right\}}} \\
& \times \exp \left\{\mathrm { i } \omega \left[\mathcal{T}\left(z ; x_{l}, \theta_{l}\right)+\frac{\sin \theta_{l}}{v}\left(x_{0}-x_{l}\right)\right.\right. \\
& \left.\left.+\frac{1}{2} \mathcal{B}\left(z, x_{l}, \theta_{l}\right) \mathcal{C}^{-1}\left(z, x_{l}, \theta_{l}\right)\left(x_{0}-x_{l}\right)^{2}\right]\right\} \\
& +\left(\vartheta_{L}^{(0)}-\vartheta_{L-1}^{(0)}\right) \sqrt{\frac{v\left(z, x_{L}\right)}{\left.\mathcal{C}\left(z, x_{L}, \theta_{L}\right) \cos \theta_{L}\right\}}} \\
& \times \exp \left\{\mathrm { i } \omega \left[\mathcal{T}\left(z ; x_{L}, \theta_{L}\right)+\frac{\sin \theta_{L}}{v}\left(x_{0}-x_{L}\right)\right.\right. \\
& \left.\left.\left.+\frac{1}{2} \mathcal{B}\left(z, x_{L}, \theta_{L}\right) \mathcal{C}^{-1}\left(z, x_{L}, \theta_{L}\right)\left(x_{0}-x_{L}\right)^{2}\right]\right\}\right]
\end{aligned}
$$

or some more sophisticated integration scheme. As we shall see, for a given $x_{0}$ only a few terms in the sum will contribute owing to the smallness of the integrand when $x$ differs significantly from $x_{0}$, i.e., the smallness of the field of a Gaussian beam away from its axis. On the other hand, for lower frequencies, the width of the beams will 
limit how near $x_{0}$ may be taken to the boundary of the computational domain.

Even though the Lagrangian formulation 28 and the Eulerian formulation 40 look quite different, they are theoretically equivalent to each other, because we are integrating over the same curve as represented by $\gamma\left(z ; \boldsymbol{x}_{s}\right)$ and $\Gamma\left(z ; \boldsymbol{x}_{s}\right)$. They are parameterized by the same 2D parameter, the takeoff direction.

In terms of numerical implementation, in the Lagrangian formulation $\gamma\left(0 ; \boldsymbol{x}_{s}\right)$ is uniformly sampled because of the uniform sampling of the takeoff angle, whereas $\gamma\left(z ; \boldsymbol{x}_{s}\right)$ is not uniformly sampled, yielding nonuniform sampling of traveltimes, as shown in Figure 1. In the Eulerian formulation $\Gamma\left(z ; \boldsymbol{x}_{s}\right)$ is uniformly sampled, yielding uniform sampling of traveltimes, as illustrated in Figure 2, implying that the resulting sampling of takeoff directions must be nonuniformly distributed in the interval $\left[-\theta_{\max }, \theta_{\max }\right]$.

The advantages of the previous Eulerian formulation are multifold. The first advantage is that we have uniform resolution of ray distribution; therefore, the Gaussian beam summation will have uniform resolution as well. The second advantage is that we can obtain wavefields excited at different sources by varying only $x_{s}$ in the summation formula 40 because all the necessary ingredients are computed already in the previous Eulerian formulation. The third advantage is that we can obtain wavefields excited at different frequencies as well by varying only $\omega$ in the summation formula 40 .

\section{NUMERICAL PROCEDURES}

\section{Discretization}

We give the beam summation algorithm for constructing wavefields.

Wavefield construction using Eulerian Gaussian Beams:

1. Discretize the computational domain using

$$
\begin{gathered}
x_{i}=x_{\min }+\left(\begin{array}{c}
i-1) \Delta x, \quad \Delta x=\frac{x_{\max }-x_{\min }}{I-1}, \\
i=1,2, \cdots, I
\end{array}\right. \\
\theta_{j}=\theta_{\min }+\begin{array}{c}
j-1) \Delta \theta, \quad \Delta \theta=\frac{\theta_{\max }-\theta_{\min }}{J-1}, \\
j=1,2, \cdots, J
\end{array} \\
z_{k}=z_{s}+(k-1) \Delta z, \quad \Delta z=\frac{z_{f}-z_{s}}{K-1}, \\
k=1,2, \cdots, K,
\end{gathered}
$$

a)

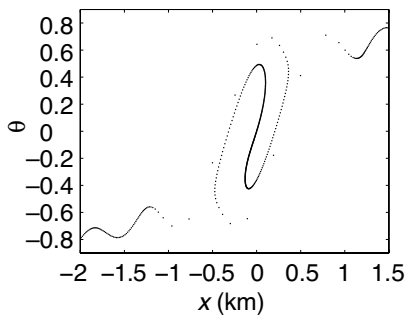

b)

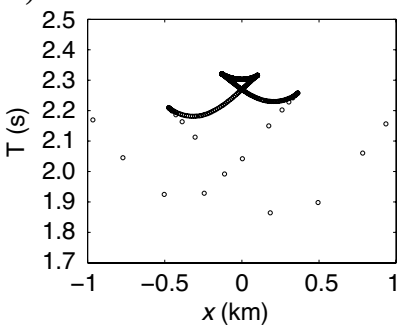

Figure 1. Sinusoidal Model, $v(z, x)=1+0.2 \sin (0.5 \pi z) \sin (3 \pi(x$ $-0.55)$ ). The curve $\gamma\left(z, x_{s}\right)=\gamma(2,0)$ by tracing 800 rays with takeoff angles uniformly sampled from $-4 \pi / 5$ to $4 \pi / 5$. (a) Arrival angles and arrival locations are not uniformly sampled; (b) the resulting traveltimes are not uniformly sampled either. and initialize all functions at $k=1$, where

$$
\begin{aligned}
\phi_{i, j, k} & =x_{i} \\
T_{i, j, k} & =0 \\
B_{i, j, k} & =i \epsilon \cos \theta_{j} \\
C_{i, j, k} & =\frac{1}{\cos \theta_{j}} \\
\psi_{i, j, k} & =\theta_{j} .
\end{aligned}
$$

2. Solve the Liouville equations according to equations 49-51 and 53. For each $i=1, \cdots, I, j=1, \cdots, J$ and $k=2, \cdots, K$, determine

$$
\begin{gathered}
\phi\left(x_{i}, \theta_{j}, z_{k}\right), \quad T\left(x_{i}, \theta_{j}, z_{k}\right), \quad B\left(x_{i}, \theta_{j}, z_{k}\right), \\
C\left(x_{i}, \theta_{j}, z_{k}\right), \quad \psi\left(x_{i}, \theta_{j}, z_{k}\right) .
\end{gathered}
$$

3. For each level $z=z_{k}, k=2, \cdots, K$, and for each physical location $x=x_{i}$, determine all $\theta_{m}^{*}$ such that $\phi\left(x_{i}, \theta_{m}^{*}, z_{k}\right)=x_{s}$ with $m$ $=1, \cdots, m(i)$. Compute the weight $\Delta \psi\left(x_{i}, \theta_{m}^{*}, k\right)$ for $m$ $=1, \cdots, m(i)$.

4. Integrate along the level set $\phi=x_{s}$ to sum up all individual Gaussian Beams to construct the wavefield.

In the above algorithm, Steps 1 and 2 can be interpreted as preprocessing steps, which are the most time consuming. Step 3 and 4 are postprocessing steps to construct the wavefield emanating from a particular point source. When we want to compute the wavefield with the same velocity model from a different point source, we only need to repeat the postprocessing step by changing the value of $x_{s}$ in the algorithm. a)

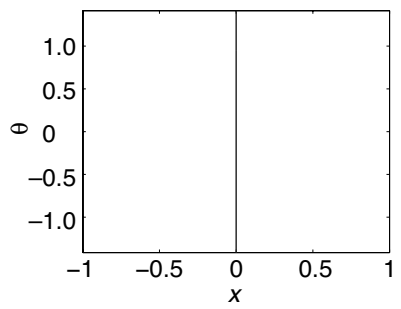

b)

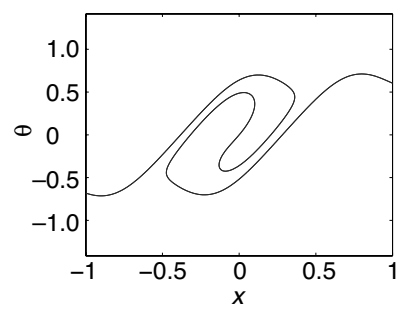

c)

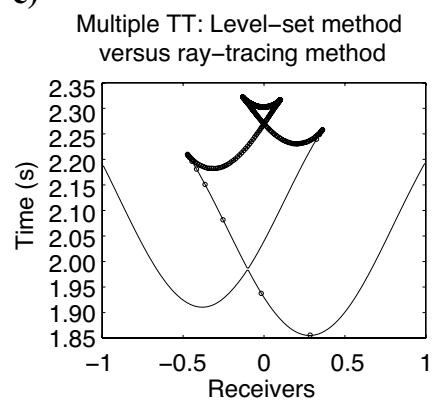

Figure 2. Sinusoidal Model. The evolution of level sets for the source location $x_{s}=0$. (a) $z=0$; (b) $z=2.0$ : arrival angles and arrival locations are uniformly sampled in the $x-\theta$ space, implying that the corresponding takeoff angles are not uniformly sampled. (c) The traveltimes are uniformly sampled as indicated by the solid line, using the level set method (Qian and Leung, 2004 and 2006). The circles are the computed solution when using the ray tracing method. 


\section{Semi-Lagrangian methods}

We solve Liouville equations 49 and 51 by a semi-Lagrangian method (Leung et al., 2004), which can be generalized to the 3D case easily; see Figure 3. Compared to finite-difference methods, semiLagrangian methods are preferred because of their ability to deal with high dimensional Liouville equations in terms of computational memory and complexity (Leung et al., 2004). We apply the method of characteristics to the equations for the level set function, the takeoff angle, and the traveltime function, which yields

$$
\frac{D \phi}{D z}=0, \quad \frac{D \psi}{D z}=0, \quad \frac{D T}{D z}=\frac{1}{v \cos \theta},
$$

where $D / D z$ is the material derivative defined by

$$
\frac{D}{D z}=\frac{\partial}{\partial z}+u \frac{\partial}{\partial x}+w \frac{\partial}{\partial \theta} \text {. }
$$

At each grid point $\left(x_{i}, \theta_{j}, z_{k}\right)$ for $i=1, \cdots, I, j=1, \cdots, J$ and $k$ $=2, \cdots, K$ in phase space, one traces backward from $z=z_{k}$ to $z=z_{s}$ $=z_{1}$ along the characteristic by integrating $d x / d z=u$ and $d \theta / d z$ $=w$ to obtain $\left(x\left(z_{s}\right), \theta\left(z_{s}\right)\right)$. For the level set equation and the takeoff angle equation, one assigns $\phi\left(x_{i}, \theta_{j}, z_{k}\right)=\phi\left(x\left(z_{s}\right), \theta\left(z_{s}\right), z_{s}\right)=x\left(z_{s}\right)$ and $\psi\left(x_{i}, \theta_{j}, z_{k}\right)=\psi\left(x\left(z_{s}\right), \theta\left(z_{s}\right), z_{s}\right)=\theta\left(z_{s}\right)$. For the traveltime equation, we use the reciprocal principle and integrate the source term $(v \cos \theta(z))^{-1}$ along the characteristics to obtain $T\left(x_{i}, \theta_{j}, z_{k}\right)$.

As for $B$ and $C$, applying the method of characteristics, we have

$$
\begin{aligned}
& \frac{D B}{D z}=-H_{x p} B-H_{x x} C \\
& \frac{D C}{D z}=H_{p p} B+H_{x p} C,
\end{aligned}
$$

with the initial conditions imposed on the level $z=z_{s}$. In this case, we do not have the reciprocal principle as for the traveltime equation anymore; we need to use the forward ray tracing to solve these quantities along the same characteristic provided by the backward ray a)

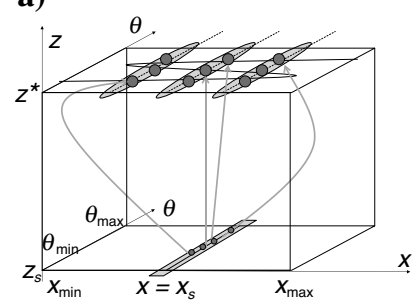

b)

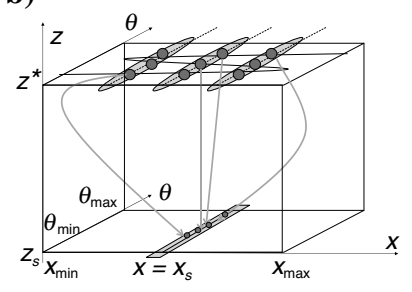

Figure 3. Lagrangian versus Semi-Lagrangian Method. (a) Lagrangian methods trace rays to an observation point starting from the source; (b) Semi-Lagrangian methods trace rays, starting from an observation point, back to the source. a) On the

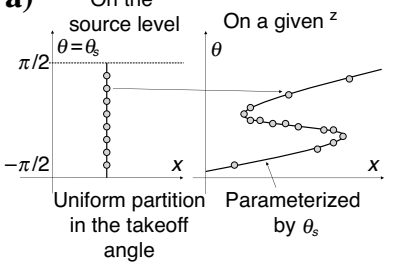

b)

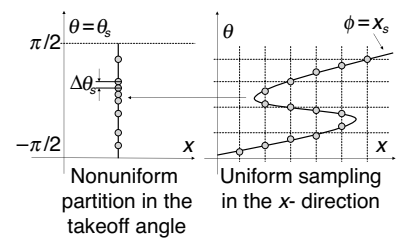

Figure 4. (a) Lagrangian versus (b) Eulerian Gaussian beam summation. tracing. This means that one first computes the ray trajectory by integrating $d x / d z=u$ and $d \theta / d z=w$ backward in $z$, and then integrates equation 62 forward in $z$ along the same characteristic.

Numerically, we adopt the second-order Stormer/Verlet scheme (Hairer et al., 2002), which preserves the symplectic structure of the Hamiltonian; certainly, one may use higher order schemes at the cost of more sophisticated implementation (Hairer et al., 2002).

\section{Wavefield construction for multiple point sources}

Consider a given source location $\left(x_{s}, z_{s}\right)$. On each level $z_{k}$, for all $x_{i}$, we determine all $\theta^{*}$ such that $\phi\left(x_{i}, \theta^{*}, z_{k}\right)=x_{s}$. Each of these points $\left(x_{i}, \theta^{*}\right)$ corresponds to one central ray which passes through the point $\left(z_{k}, x_{i}\right)$ in the physical space at the arrival angle $\theta^{*}$; correspondingly, we can determine the traveltime $T$, the takeoff angle $\psi, B$, and $C$ for this ray by interpolating the available $T, \psi, B$, and $C$ at $\left(z_{k}, x_{i}, \theta^{*}\right)$. This information defines one particular Gaussian beam centered at $\left(z_{k}, x_{i}\right)$. Next, at given $z_{k}$ we collect all arrival rays and sort them in the ascending order of the take-off angle. The contribution from all Gaussian beams to a physical location $\left(z_{k}, x_{i}\right)$ can then be computed by integrating equation 54 (see Figure 4).

\section{Computing the weight for each individual Gaussian beam}

1) For a given $z=z_{k}$ and for all $i=1, \cdots, I$
a)
Given $\phi\left(x_{i}, \theta_{j}, z_{k}\right)$ for all $j=1, \cdots, J$, determine all $\theta_{m}^{*}$ such that $\phi\left(x_{i}, \theta_{m}^{*}, z_{k}\right)=x_{s}$ for $m=1, \cdots, m(i)$.
b) Interpolate and obtain $\psi\left(x_{i}, \theta_{m}^{*}, z_{k}\right)$ for $m=1, \cdots, m(i)$.

2) For all $i=1, \cdots, I$ and $m=1, \cdots, m(i)$

a) Among all points $\left(x_{i \pm 1}, \theta_{n_{1}}^{*}, z_{k}\right)$ and $\left(x_{i}, \theta_{n_{2}}^{*}, z_{k}\right)$ for $n_{1}$ $=1, \cdots, m(i \pm 1)$ and $n_{2}=1, \cdots, m(i)$ and $n_{2} \neq m$, determine two points which have values $\psi$ closest to $\psi\left(x_{i}, \theta_{m}^{*}, z_{k}\right)$.

b) Set $\Delta \psi\left(x_{i}, \theta_{m}^{*}, z_{k}\right)$ equal to the mean of these two differences.

Equation 54 has two different interpretations, which yield two different algorithms for summing up all Gaussian Beams.

1) For each observation point $\left(x_{i}, z_{k}\right)$, one first searches in a neighborhood centered at this observation point for all Gaussian beams and sums the contribution from each of these beams according to their corresponding weights using equation 54 , as shown in Figure 5a;

2) For each point $\left(x_{i}, z_{k}\right)$, one first determines all Gaussian beams which pass through it. For each of these beams, one computes its contribution to the neighboring observation points, as shown in Figure 5b.

Numerically, the second interpretation yields a more efficient summation algorithm than the first interpretation. Therefore, we only give the algorithm based on the second interpretation.

\section{Wavefield construction algorithm}

1) Initialize $\operatorname{Re}(U)_{i, k}=\operatorname{Im}(U)_{i, k}=0$

2) For $i=1, \cdots, I$ 
3) For $k=1, \cdots, K$

a) Given $\phi\left(x_{i}, \theta_{j}, z_{k}\right)$ for all $j=1, \cdots, J$, determine all $\theta^{*}$ such that $\phi\left(x_{i}, \theta^{*}, z_{k}\right)=x_{s}$.

b) For each $\theta^{*}$ and for $i^{\prime}=1, \cdots, I$

i. Compute

$$
\begin{aligned}
& \operatorname{Re}(U)_{i^{\prime}, k}=\operatorname{Re}(U)_{i^{\prime}, k} \\
& +\frac{1}{4 \pi} \Delta \psi\left(x_{i}, \theta^{*}, z_{k}\right) \\
& \times\left|A\left(x_{i}, \theta^{*}, z_{k}\right)\right| \exp \left[-\frac{\omega}{2}\right. \\
& \left.\times \operatorname{Im}\left(\frac{B\left(x_{i}, \theta^{*}, z_{k}\right)}{C\left(x_{i}, \theta^{*}, z_{k}\right)}\right)\left(x_{i^{\prime}}-x_{i}\right)^{2}\right] \\
& \times \cos \left\{\omega \left(T\left(x_{i}, \theta^{*}, z_{k}\right)\right.\right. \\
& +\frac{\sin \theta^{*}}{v\left(x_{i}, z_{k}\right)}\left(x_{i^{\prime}}-x_{i}\right) \\
& \left.+\frac{1}{2} \operatorname{Re}\left[\frac{B\left(x_{i}, \theta^{*}, z_{k}\right)}{C\left(x_{i}, \theta^{*}, z_{k}\right)}\right]\left(x_{i^{\prime}}-x_{i}\right)^{2}\right) \\
& \left.+\frac{\pi}{2}-\frac{1}{2} \operatorname{Arg}\left[C\left(x_{i}, \theta^{*}, z_{k}\right)\right]\right\} \\
& \operatorname{Im}(U)_{i^{\prime}, k}=\operatorname{Im}(U)_{i^{\prime}, k}+\frac{1}{4 \pi} \Delta \psi\left(x_{i}, \theta^{*}, z_{k}\right) \\
& \times\left|A\left(x_{i}, \theta^{*}, z_{k}\right)\right| \exp \left[-\frac{\omega}{2}\right. \\
& \left.\times \operatorname{Im}\left(\frac{B\left(x_{i}, \theta^{*}, z_{k}\right)}{C\left(x_{i}, \theta^{*}, z_{k}\right)}\right)\left(x_{i^{\prime}}-x_{i}\right)^{2}\right] \\
& \times \sin \left\{\omega \left(T\left(x_{i}, \theta^{*}, z_{k}\right)\right.\right. \\
& +\frac{\sin \theta^{*}}{v\left(x_{i}, z_{k}\right)}\left(x_{i^{\prime}}-x_{i}\right) \\
& \left.+\frac{1}{2} \operatorname{Re}\left[\frac{B\left(x_{i}, \theta^{*}, z_{k}\right)}{C\left(x_{i}, \theta^{*}, z_{k}\right)}\right]\left(x_{i^{\prime}}-x_{i}\right)^{2}\right) \\
& \left.+\frac{\pi}{2}-\frac{1}{2} \operatorname{Arg}\left[C\left(x_{i}, \theta^{*}, z_{k}\right)\right]\right\}
\end{aligned}
$$

\section{c) End for}

\section{4) End for}

\section{5) End for}

In the algorithm, $i^{\prime}=1, \cdots, I$ in Step $3 \mathrm{~b}$ can be replaced by a local neighborhood $i^{\prime}=i-\epsilon_{i}, \cdots, i+\epsilon_{i}$ whenever $\epsilon_{i}$ is chosen to be large enough.

\section{NUMERICAL EXPERIMENTS}

In the following numerical examples, we use $257=2^{8}+1$ grid points in each $x$-, $\theta$-, and $z$-direction. The computational domain is $[x, \theta, z] \in[-1,1] \times\left[-\theta_{\max }, \theta_{\max }\right] \times[0,2]$. We take $\theta_{\max }=9 \pi / 20$ for the constant velocity model and $\theta_{\max }=8 \pi / 20$ for the waveguide model. A grayscale plot of the waveguide model is shown in Figure 6.

We choose the initial beam width to be $\operatorname{Im}(B(0))=\epsilon \cos \theta_{s}$ $=\cos \theta_{s}$, and we are not going to optimize the beam width in the summation process. Although narrow beams combined with an appropriate window function will yield a more efficient summation algorithm, generally it is not an easy task to specify an a priori beam width which will be narrow throughout the computation.

Because our Gaussian beam formulation is based on global Cartesian coordinates, and the asymptotic solution does not depend on the initial beam width, in this work we make the above choice of the initial beam width and sum up all possible contributions from each beam at each observation point. On the other hand, our examples demonstrate that one may not be able to get accurate solutions if one chooses a summation window to be too narrow at the source because the narrow beam will become wider along the ray in general. a)

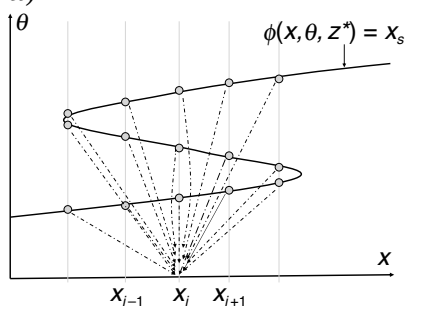

b)

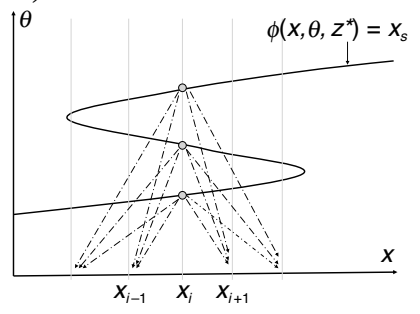

Figure 5. Eulerian Gaussian beam summation. Each circle on the level set $\phi=x_{s}$ denotes a particular central ray passing through a physical location $\left(x_{i}, z_{k}\right)$, which represents one particular Gaussian beam. Dashed lines denote the directions of the contribution. (a) Each observation point receives contributions from all the possible beams in its neighborhood; (b) each point $\left(x_{i}, z_{k}\right)$ owns several beams, and every neighboring observation point receives contribution from each of these beams.

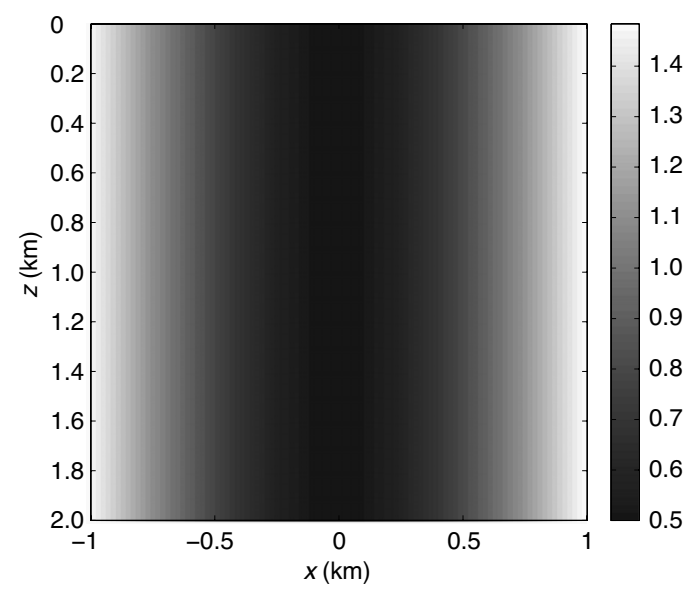

Figure 6. The grayscale plots of the velocity field of the waveguide model. 
a)

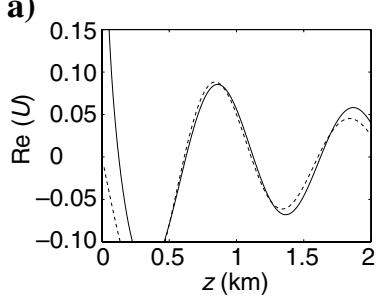

c)

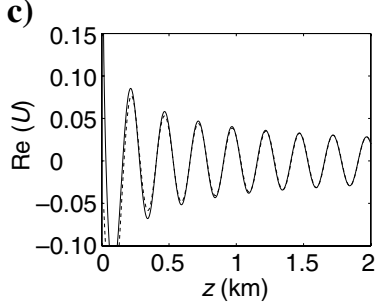

e)

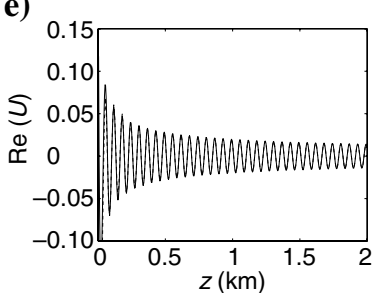

b)

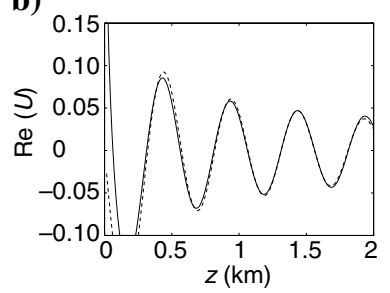

d)

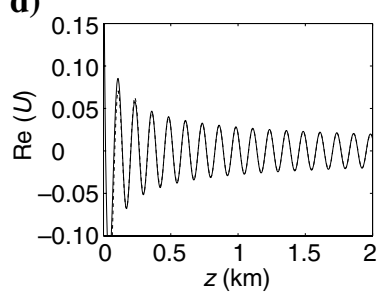

f)

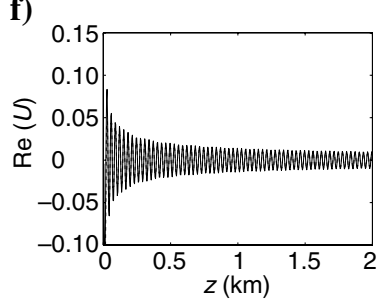

Figure 7. Constant Model, source at $x_{s}=z_{s}=0 \mathrm{~km}$. Cross sections of $\operatorname{Re}(U)$ along $x=0 \mathrm{~km}$ for (a) $\omega=2 \pi$, (b) $\omega=4 \pi$, (c) $\omega=8 \pi$, (d) $\omega=16 \pi$, (e) $\omega=32 \pi$, and (f) $\omega=64 \pi$. The solid lines are the asymptotic ray theory solution; the dashed curves are the computed solution using the proposed Gaussian beam approach.

a)

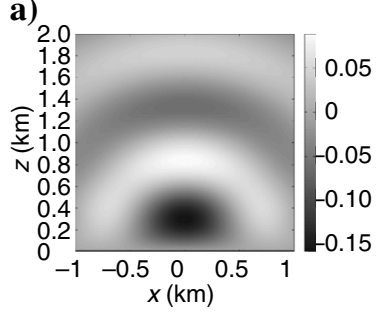

c)

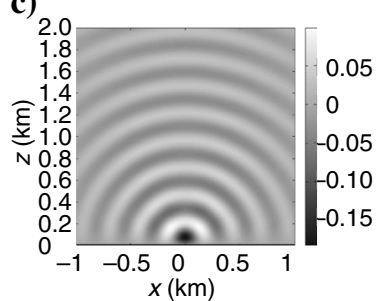

e)

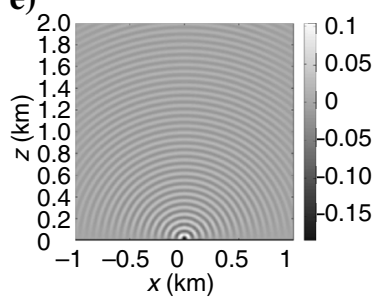

b)

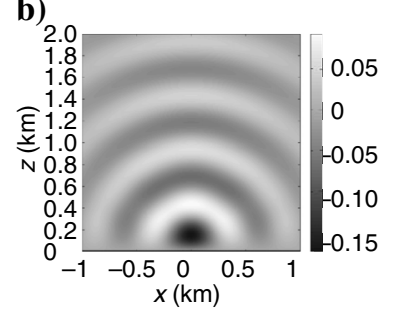

d)

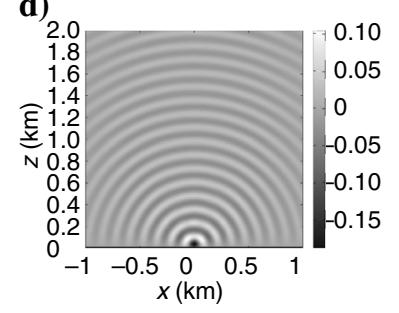

f)

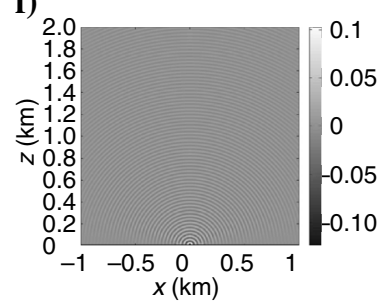

Figure 8. Constant Model, source at $x_{s}=z_{s}=0 \mathrm{~km}$. The real part of the wavefield for (a) $\omega=2 \pi$, (b) $\omega=4 \pi$, (c) $\omega=8 \pi$, (d) $\omega=16 \pi$, (e) $\omega=32 \pi$, and (f) $\omega=64 \pi$.

\section{Constant model}

We take $v(z, x) \equiv 1$. The asymptotic ray theory (ART) solution of the wavefield is the asymptotic expansion of the Hankel function, which is the exact solution of the Helmholtz equation with a point source (see Appendix B). We will use this exact solution to calibrate the numerical solution based on Eulerian Gaussian beams.

Next, we construct the time harmonic wave fields with different frequencies by using Eulerian Gaussian beams. Figures 7-10 show the results when no window function is used in the summation process, meaning that in the wavefield construction algorithm we set the local neighborhood to be infinity.

In the low frequency regime, for example, when $\omega=2 \pi$, the wavefield shown in Figure 7 and the phase shown in Figures 9 and 10 do not agree very well with the exact solution, which is expected because we are approximating the wave equation in the high frequency regime. In the high frequency regime, as we can see from Figure 7, our Gaussian beam solution matches the ART solution very well. Because the asymptotes of the Hankel function for $\omega \rightarrow \infty$ and $\sqrt{x^{2}+z^{2}}$ $\rightarrow \infty$ coincide, Figure 7 also illustrates that for fixed $\omega$, the Gaussian beam solution becomes more accurate as $\sqrt{x^{2}+z^{2}} \rightarrow \infty$.

On the other hand, finite-difference modeling for directly solving Helmholtz equations usually requires 10 to 12 mesh points per wavelength to resolve wave propagation well, and the optimal number of mesh points per wavelength is 3 to 5 . When $\omega=64 \pi$, the a)

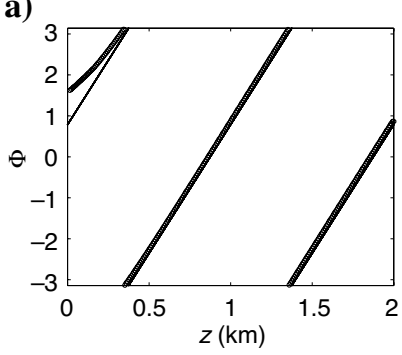

c)

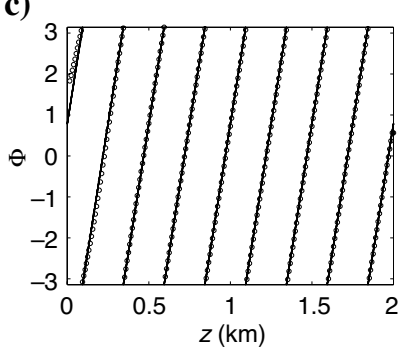

e)

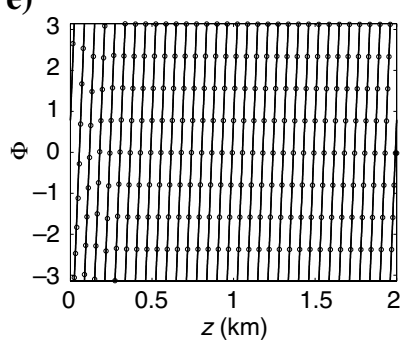

b)

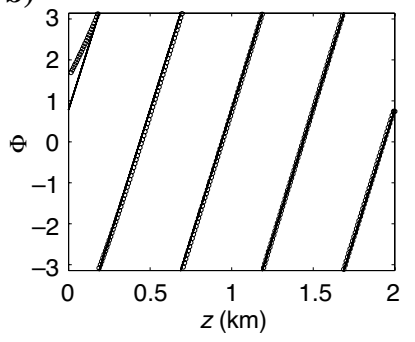

d)

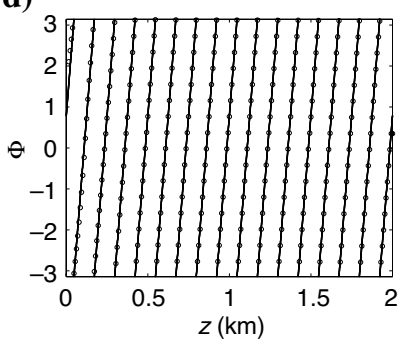

f)

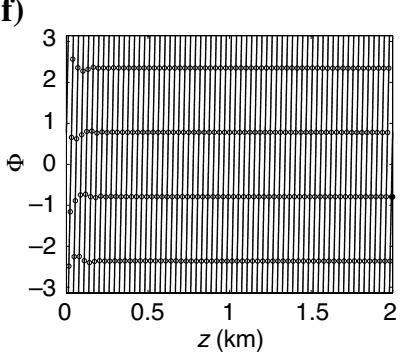

Figure 9. Constant Model, source at $x_{s}=z_{s}=0 \mathrm{~km}$. Cross section of the phase along $x=0 \mathrm{~km}$ with (a) $\omega=2 \pi$, (b) $\omega=4 \pi$, (c) $\omega=8 \pi$, (d) $\omega=16 \pi$, (e) $\omega=32 \pi$, and (f) $\omega=64 \pi$. The solid lines are the asymptotic ray theory solution; the circles are the computed solution using the proposed Gaussian beam approach. 
wavelength $\lambda=2 \pi / \omega=1 / 32$; thus, the number of waves from $z$ $=0$ to $z=2$ is roughly 64 . Because we have 256 mesh points along the $z$ direction, there are roughly 4 mesh points per wavelength, which is almost optimal (see Figure 7f).

Figure 11 shows that the Gaussian beam solution might not be accurate if one chooses the summation window of the beam that is too narrow; here, the half width of the window is $3 \Delta x$.

\section{Waveguide model}

The velocity function is

$$
v(z, x)=3-2.5 \exp \left(-0.5 x^{2}\right) .
$$

Ray tracing indicates that with appropriate source locations this model yields cusp-type caustics; traditional ray theory predicts infinite amplitude at caustics, whereas Gaussian beam theory predicts finite amplitude there. Therefore, we use this model to test the validity of Gaussian beam theory.

Figures 12 and 13 show the time harmonic wavefields excited at different sources with respect to different frequencies. As we can see, the wavefields become more concentrated as the frequency increases. At the same time, at caustics the wavefield stays finite, as it should. Figure 14 indicates that inside the cusp the wavefield becomes stronger. a)

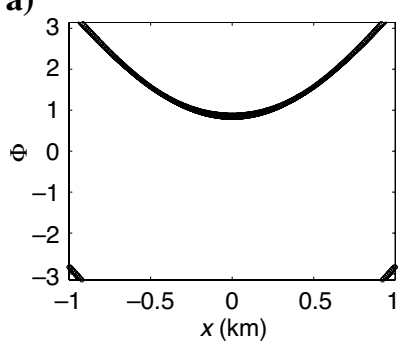

c)

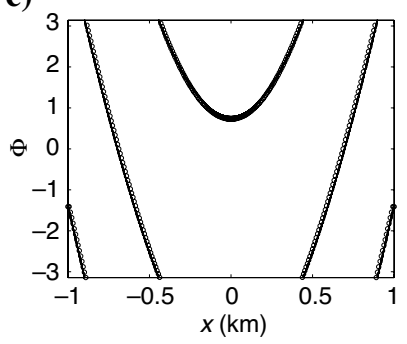

e)

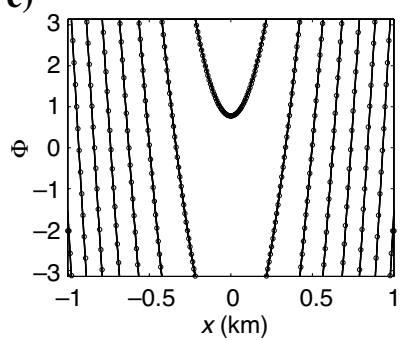

b)

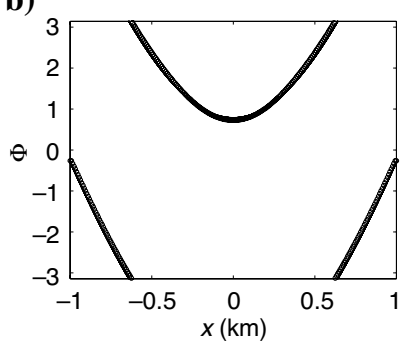

d)

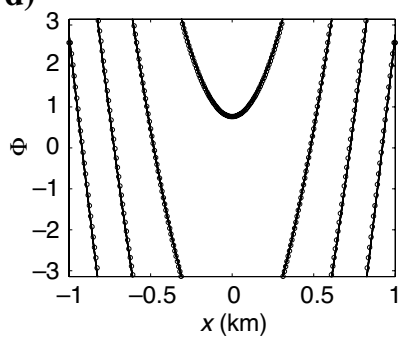

f)

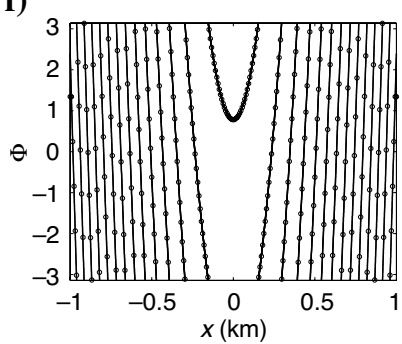

a)

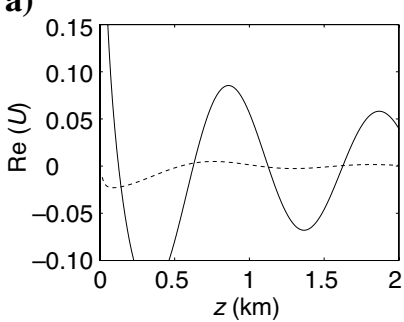

c)

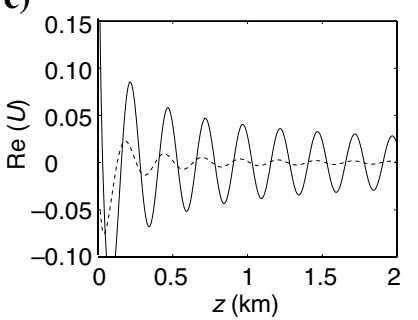

e)

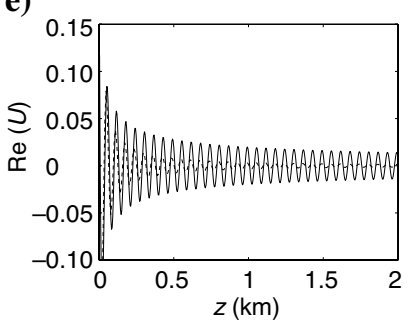

b)

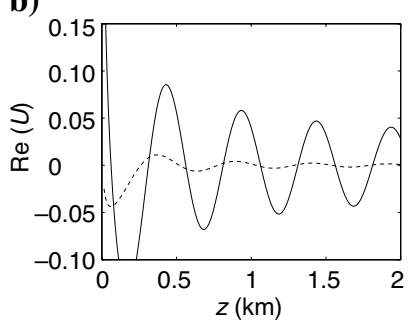

d)

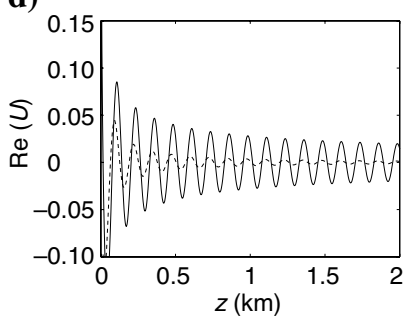

f)

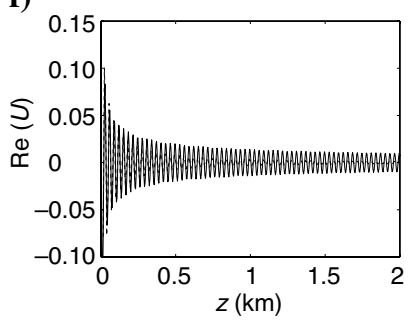

Figure 11. Constant Model, source at $x_{s}=z_{s}=0 \mathrm{~km}$ and $\epsilon_{i}=3 \Delta x$. Cross sections of $\operatorname{Re}(U)$ along $x=0 \mathrm{~km}$ for (a) $\omega=2 \pi$, (b) $\omega=4 \pi$, (c) $\omega=8 \pi$, (d) $\omega=16 \pi$, (e) $\omega=32 \pi$, and (f) $\omega=64 \pi$. The solid lines are the asymptotic ray theory solution; the dashed curves are the computed solution using the proposed Gaussian beam approach. One can compare with Figure 7 to conclude that the accuracy in the solution depends on the summation window width of the Gaussian beam. a)

c)

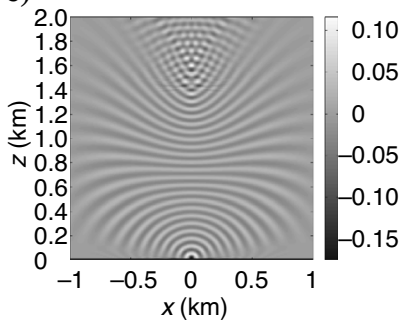

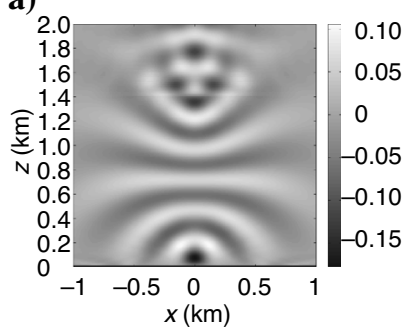

b)

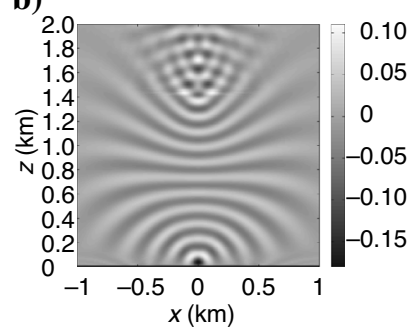

d)

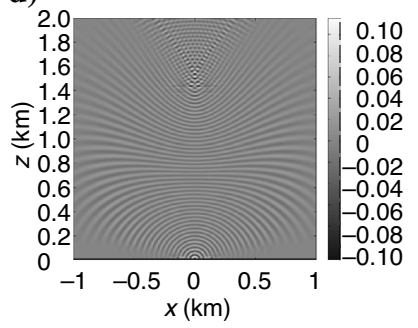

Figure 10. Constant Model, source at $x_{s}=z_{\mathrm{s}}=0 \mathrm{~km}$. Cross section of the phase along $z=2 \mathrm{~km}$ with (a) $\omega=2 \pi$, (b) $\omega=4 \pi$, (c) $\omega$ $=8 \pi$, (d) $\omega=16 \pi$, (e) $\omega=32 \pi$, and (f) $\omega=64 \pi$. The solid lines are the asymptotic ray theory solution; the circles are the computed solution using the proposed Gaussian beam approach.

Figure 12. Waveguide Model, source at $x_{s}=z_{s}=0 \mathrm{~km}$. The real part of the wavefield using (a) $\omega=4 \pi$, (b) $\omega=8 \pi$, (c) $\omega=16 \pi$, and (d) $\omega=32 \pi$. 

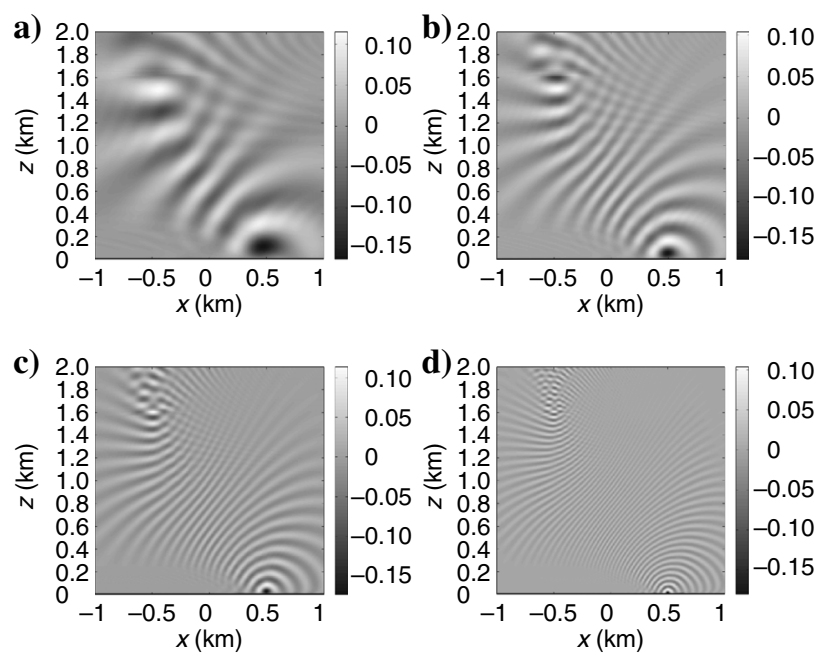

Figure 13. Waveguide Model, source at $x_{s}=0.5 \mathrm{~km}$ and $z_{s}=0 \mathrm{~km}$. The real part of the wavefield using (a) $\omega=4 \pi$, (b) $\omega=8 \pi$, (c) $\omega$ $=16 \pi$, and $(\mathrm{d}) \omega=32 \pi$.
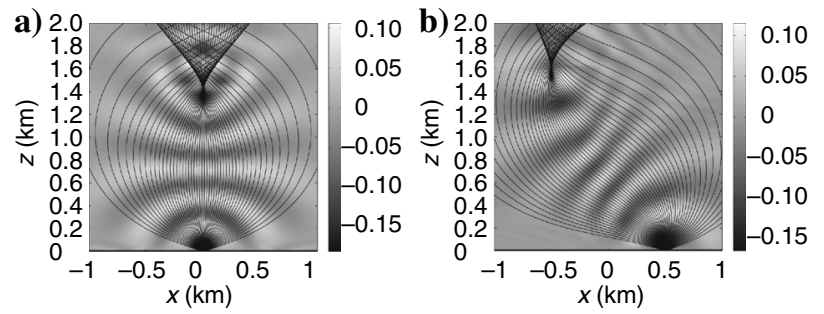

Figure 14. Waveguide Model, $\omega=8 \pi$. The real part of the wavefield overlaid with rays. (a) Source at $x_{s}=0 \mathrm{~km}$ and $z_{s}=0 \mathrm{~km}$. (b) Source at $x_{s}=0.5 \mathrm{~km}$ and $z_{s}=0 \mathrm{~km}$.

\section{CONCLUSIONS}

The traditional Gaussian beam summation method is based on Lagrangian ray tracing and local ray-centered coordinates. We propose a new Eulerian formulation of Gaussian beam theory which adopts global Cartesian coordinates, level sets, and Liouville equations, yielding uniformly distributed Eulerian traveltimes and amplitudes in phase space simultaneously for multiple sources. The time harmonic wavefield can be constructed by summing up Gaussian beams with ingredients provided by the new Eulerian formulation. Numerical experiments indicate that the Gaussian beam summation method yields accurate asymptotic wavefields even at caustics.

The conventional Gaussian beam summation method can be derived from the proposed method. The new method offers three advantages: uniform resolution of ray distribution, so that the Gaussian beam summation will have uniform resolution as well; the ability to obtain wavefields excited at different sources by varying only source locations in the summation formula; and the ability to obtain wavefields excited at different frequencies by varying only frequencies in the summation formula.

In future work, we will systematically compare our new method to the traditional Gaussian beam summation method and develop Gaussian beam migration methods based on this new formulation.

\section{LIST OF SYMBOLS}

$$
\begin{aligned}
& n=\text { the dimension of the full } \\
& \bar{n}=n-1=\text { the dimension of the } \\
& \widetilde{\boldsymbol{x}}=\left(z, x_{1}, \ldots, x_{\bar{n}}\right)=(z, \boldsymbol{x})=\text { full position vector } \\
& r=\sqrt{z^{2}+x^{2}}=\text { special (depth) coordinate } \\
& \epsilon>0=\text { parameter related to beam } \\
& \text { width } \\
& a=1+\mathrm{i} \epsilon v_{0} r=\text { recurring combination } \\
& \text { introduced for brevity } \\
& \boldsymbol{x}=\left(x_{1}, \ldots, x_{\bar{n}}\right)=\text { other (horizontal) coordinates } \\
& \boldsymbol{X}\left(z, \boldsymbol{x}_{s}, \boldsymbol{\nu}_{s}\right)=\text { value of } \boldsymbol{x} \text { along the } x \boldsymbol{\nu} \text {-ray } \\
& \text { through }\left(0, \boldsymbol{x}_{s}, \boldsymbol{\nu}_{s}\right) \\
& \boldsymbol{\mathcal { X }}^{(0)}(z, \boldsymbol{x}, \boldsymbol{\nu})=\text { initial value of } \boldsymbol{x} \text { at } z=0 \text { for } \\
& \text { the } x \boldsymbol{\nu} \text {-ray through }(z, x, \nu) \\
& \boldsymbol{p}=\left(p_{1}, \ldots, p_{\bar{n}}\right)=\boldsymbol{\nabla} \tau=\text { horizontal slowness } \\
& \boldsymbol{P}\left(z, \boldsymbol{x}_{s}, \boldsymbol{\nu}_{s}\right)=\text { value of } \boldsymbol{p} \text { along the } x \boldsymbol{\nu} \text {-ray } \\
& \text { through }\left(0, \boldsymbol{x}_{s}, \boldsymbol{\nu}_{s}\right) \\
& \widetilde{\boldsymbol{p}}=\left(p_{0}, p_{1}, \ldots, p_{\bar{n}}\right)=\left(p_{0}, \boldsymbol{p}\right)=\tilde{\boldsymbol{\nabla}} \tau=\text { full slowness vector } \\
& \widetilde{\boldsymbol{t}}=\left(t_{0}, t_{1}, \ldots, t_{\bar{n}}\right)=\widetilde{\boldsymbol{p}} /|\tilde{\boldsymbol{p}}|=\boldsymbol{v p}=\text { unit tangent to the ray, also } \\
& \text { ray direction } \\
& \begin{aligned}
\boldsymbol{t}=\left(t_{1}, \ldots, t_{\bar{n}}\right) & =\text { horizontal components of } \widetilde{\boldsymbol{t}} \\
t_{0}=\cos \theta=\sqrt{1-t_{1}^{2}-\ldots-t_{\bar{n}}^{2}}=\sqrt{1-\boldsymbol{t}^{2}} & =\theta \text { is angle between slowness }
\end{aligned} \\
& \text { and } z \text {-direction } \\
& \boldsymbol{v}=\boldsymbol{t} / t_{0}=\boldsymbol{p} / p_{0}=\text { horizontal direction } \\
& \text { parameters } \\
& \boldsymbol{N}\left(z, \boldsymbol{x}_{s}, \boldsymbol{v}_{s}\right)=\text { value of } \boldsymbol{v} \text { along the } x \boldsymbol{\nu} \text {-ray } \\
& \text { through }\left(0, \boldsymbol{x}_{s}, \boldsymbol{v}_{s}\right) \\
& \mathcal{N}^{(0)}(z, \boldsymbol{x}, \boldsymbol{v})=\text { initial value for } z=0 \text { of } \boldsymbol{v} \text { for } \\
& \text { the } x \nu \text {-ray through }(z, x, v) \\
& \boldsymbol{\alpha}=\left(\alpha_{1}, \ldots, \alpha_{\bar{n}}\right)=\text { right cross-sectional } \\
& \text { parameters of a beam } \\
& \widetilde{\boldsymbol{v}}=(1, \boldsymbol{v})=\left(\nu_{0}, \nu_{1}, \ldots, \nu_{\bar{n}}\right)=\text { full direction parameter vector } \\
& \mu=\sqrt{1+\boldsymbol{v}^{2}}=1 / \cos \theta=\text { reciprocal direction cosine } \\
& \boldsymbol{\nabla}=\left(\partial_{x_{1}}, \ldots, \partial_{x_{\bar{n}}}\right)=\text { horizontal gradient } \\
& \tilde{\nabla}=\left(\partial_{x_{0}}, \partial_{x_{1}}, \ldots, \partial_{x_{\bar{n}}}\right)=\text { full gradient } \\
& \tau=\text { travel time } \\
& \boldsymbol{T}\left(z, \boldsymbol{x}_{s}, \boldsymbol{v}_{s}\right)=\text { value of } \tau \text { along the } x \nu \text {-ray } \\
& \text { through }\left(0, \boldsymbol{x}_{s}, \boldsymbol{v}_{s}\right) \\
& \mathcal{T}(z, \boldsymbol{x}, \boldsymbol{v})=\text { value of } \tau \text { for the } x \boldsymbol{\nu} \text {-ray } \\
& \text { through }(z, \boldsymbol{x}, \boldsymbol{v}) \\
& \omega=\text { frequency } \\
& \mathrm{i} \omega \tau=\text { phase } \\
& \boldsymbol{C}\left(z, \boldsymbol{x}_{s}, \boldsymbol{v}_{s}\right)=\partial \boldsymbol{x} / \partial \boldsymbol{\alpha}=\text { variation of ray position } \\
& \text { along the } x \nu \text {-ray through } \\
& \left(0, \boldsymbol{x}_{s}, \boldsymbol{v}_{s}\right) \\
& \mathcal{C}(z, \boldsymbol{x}, \boldsymbol{v})=\partial \boldsymbol{x} / \partial \boldsymbol{\alpha}=\text { variation of ray position } \\
& \text { along the } x \nu \text {-ray through } \\
& (z, \boldsymbol{x}, \boldsymbol{v}) \\
& \boldsymbol{B}\left(z, \boldsymbol{x}_{s}, \boldsymbol{v}_{s}\right)=\partial \boldsymbol{p} / \partial \boldsymbol{\alpha}=\text { variation of ray slowness } \\
& \text { along the } x \nu \text {-ray through } \\
& \left(0, \boldsymbol{x}_{s}, \boldsymbol{v}_{s}\right) \\
& \mathcal{B}(z, \boldsymbol{x}, \boldsymbol{v})=\partial \boldsymbol{p} / \partial \boldsymbol{\alpha}=\text { variation of ray slowness } \\
& \text { along the } x \nu \text {-ray through } \\
& (z, x, v) \\
& \boldsymbol{M}=\boldsymbol{B} \boldsymbol{C}^{-1}=\partial \boldsymbol{p} / \partial \boldsymbol{x}=\tau_{\boldsymbol{x}}=\text { second horizontal derivatives } \\
& \text { of traveltime }
\end{aligned}
$$




$$
\begin{aligned}
\boldsymbol{Q}_{\|}=\frac{\boldsymbol{v} \boldsymbol{v}^{T}}{\boldsymbol{v}^{2}},= & \text { projection onto the vector } \boldsymbol{v} \\
& \text { in } R^{\bar{n}} \\
\boldsymbol{Q}_{\perp}=\boldsymbol{I}-\boldsymbol{Q}_{\|}= & \text {projection onto the plane } \\
& \text { orthogonal to } \boldsymbol{v} \text { in } R^{\bar{n}}
\end{aligned}
$$

\section{ACKNOWLEDGMENT}

Jianliang Qian would like to acknowledge the financial support of the National Science Foundation (Grant no. 0542174) and the Air Force Office of Scientific Research (Grant no. FA9550-04-1-0143).

\section{APPENDIX A}

\section{THE SECOND DERIVATIVE OF TRAVELTIME AND AMPLITUDE}

\section{The second derivative of traveltime}

Lemma Under the above assumptions, $\operatorname{det}\left\{\boldsymbol{C}\left(z ; \boldsymbol{x}_{s}, \boldsymbol{t}_{s}\right)\right\} \neq 0$ for any $z$, and $\operatorname{Im}\left(\boldsymbol{B} \boldsymbol{C}^{-1}\right)$ is real, symmetric, positive definite for all $z$.

Proof We use the same technique as in Tanushev et al. (2007) to prove this lemma. Let $\left(\boldsymbol{B}_{1}(z), \boldsymbol{C}_{1}(z)\right)$ and $\left(\boldsymbol{B}_{2}(z), \boldsymbol{C}_{2}(z)\right)$ be two solutions of the DRT system with different initial conditions along the same ray trajectory defined by the ray tracing system. Then the following Wronskian function

$$
\boldsymbol{W}(z)=\boldsymbol{C}_{1}(z)^{T} \boldsymbol{B}_{2}(z)-\boldsymbol{B}_{1}(z)^{T} \boldsymbol{C}_{2}(z)
$$

is constant along the ray; this is established by differentiating $\boldsymbol{W}(z)$ in $z$. In what follows, we use the superscript ${ }^{(T)}$ to denote the transpose of a matrix, an overbar (-) to denote the complex conjugate, and ${ }^{(\dagger)}$ to denote the conjugate transpose.

Now suppose that $\boldsymbol{C}(z) \boldsymbol{v}=\mathbf{0}$ for some $z$ and some nonzero vector $\boldsymbol{v}$. Then applying the Wronskian identity to the two solutions, $(\overline{\boldsymbol{B}(z)}, \overline{\boldsymbol{C}(z)})$ and $(\boldsymbol{B}(z), \boldsymbol{C}(z))$, we have

$$
\begin{aligned}
0= & \boldsymbol{v}^{\dagger}\left[\boldsymbol{C}(z)^{\dagger} \boldsymbol{B}(z)-\boldsymbol{B}(z)^{\dagger} \boldsymbol{C}(z)\right] \boldsymbol{v}=\boldsymbol{v}^{\dagger}\left[\boldsymbol{C}(0)^{\dagger} \boldsymbol{B}(0)\right. \\
& \left.-\boldsymbol{B}(0)^{\dagger} \boldsymbol{C}(0)\right] \boldsymbol{v}=2 \mathrm{i} \boldsymbol{\epsilon} \boldsymbol{v}^{\dagger} \boldsymbol{N}_{s} \boldsymbol{v} \neq 0,
\end{aligned}
$$

because $\boldsymbol{\epsilon}$ is positive and $\boldsymbol{N}_{s}=\boldsymbol{I}+\boldsymbol{\nu} \boldsymbol{v}^{T}$ is real, symmetric, and positive definite. Thus, we have a contradiction. Therefore, $\boldsymbol{C}(z)$ is nonsingular, and we may form $\boldsymbol{M}(z)=\boldsymbol{B}(z) \boldsymbol{C}(z)^{-1}$. Now let us verify that $\boldsymbol{M}(z)=\boldsymbol{B}(z) \boldsymbol{C}(z)^{-1}$ is symmetric. But $\boldsymbol{C}(0)=\boldsymbol{Q}_{\perp}+\mu \boldsymbol{Q}_{\|}$and $\boldsymbol{B}(0)$ $=\mathrm{i} \epsilon\left[\boldsymbol{Q}_{\perp}+(1 / \mu) \boldsymbol{Q}_{\|}\right]$are both symmetric and commute with each other, so by applying the Wronskian identity to $(\boldsymbol{B}, \boldsymbol{C})$ and itself, we get

$$
\begin{aligned}
\mathbf{0} & =\boldsymbol{C}(0)^{T} \boldsymbol{B}(0)-\boldsymbol{B}(0)^{T} \boldsymbol{C}(0)=\boldsymbol{C}(z)^{T} \boldsymbol{B}(z)-\boldsymbol{B}(z)^{T} \boldsymbol{C}(z) \\
& =\boldsymbol{C}(z)^{T}\left[\boldsymbol{M}(z)-\boldsymbol{M}(z)^{T}\right] \boldsymbol{C}(z) .
\end{aligned}
$$

Hence, because $\boldsymbol{C}(z)$ is nonsingular, $\boldsymbol{M}(z)$ is (complex) symmetric.

Next notice that $\boldsymbol{C}(0)^{\dagger} \boldsymbol{B}(0)=\mathrm{i} \boldsymbol{\epsilon}$, so that

$$
\begin{aligned}
2 \mathrm{i} \epsilon \boldsymbol{C}(z)^{-\dagger} \boldsymbol{I} \boldsymbol{C}(z)^{-1} & =\boldsymbol{C}(z)^{-\dagger}\left[\boldsymbol{C}(0)^{\dagger} \boldsymbol{B}(0)-\boldsymbol{B}(0)^{\dagger} \boldsymbol{C}(0)\right] \boldsymbol{C}(z)^{-1}, \\
& =\boldsymbol{C}(z)^{-\dagger}\left[\boldsymbol{C}(z)^{\dagger} \boldsymbol{B}(z)-\boldsymbol{B}(z)^{\dagger} \boldsymbol{C}(z)\right] \boldsymbol{C}(z)^{-1}, \\
& =\left[\boldsymbol{M}(z)-\boldsymbol{M}(z)^{\dagger}\right]=2 \mathrm{i} \operatorname{Im}\{\boldsymbol{M}(z)\} . \quad(\mathrm{A}-4)
\end{aligned}
$$

Therefore, comparing the first and last members of this string of equalities, we see that $\operatorname{Im}\{\boldsymbol{M}(z)\}=\operatorname{Im}\left\{\boldsymbol{B} \boldsymbol{C}^{-1}\right\}$ is positive definite be- cause $\epsilon C(z)^{-\dagger} N_{s} C(z)^{-1}$ is, the eigenvalues of the real symmetric matrix $\operatorname{Im}\{\boldsymbol{M}(0)\}$ being 1 and $1 /\left(1+\boldsymbol{v}^{2}\right)$.

\section{Transport of amplitude}

We have the following transport equation for amplitude $A$ $=A\left(z, \boldsymbol{x} ; \boldsymbol{x}_{s}, \boldsymbol{v}_{s}\right)$, which represents energy flux conservation along tubes of rays:

$$
\widetilde{\nabla} \cdot\left(A^{2} \widetilde{\nabla} \tau\right)=0
$$

It can be rewritten as

$$
\begin{gathered}
\frac{\partial}{\partial z}(\log A) \tau_{z}+\frac{\partial}{\partial x_{1}}(\log A) \tau_{x_{1}}+\ldots+\frac{\partial}{\partial x_{\bar{n}}}(\log A) \tau_{\bar{n}} \\
+\frac{1}{2}\left(\tau_{z z}+\tau_{x_{1} x_{1}}+\ldots+\tau_{x_{\bar{n}} x_{\bar{n}}}\right)=0
\end{gathered}
$$

which also is almost immediate from equation A-10. Thus, along a paraxial ray, i.e., one for which $\tau_{z}>0$, we have

$$
\begin{aligned}
\frac{\partial}{\partial z}(\log A)+\boldsymbol{v}^{T} \boldsymbol{\nabla} \log A+\frac{1}{2 \tau_{z}}\left(\tau_{z z}+\nabla^{2} \tau\right) & =0, \\
\frac{d}{d z}(\log A)+\frac{1}{2 \tau_{z}}\left(\tau_{z z}+\nabla^{2} \tau\right) & =0 .
\end{aligned}
$$

To obtain the expression for $\tau_{z z}+\nabla^{2} \tau$ along a ray, we integrate the left side of equation A-7 over the volume contained by the ray tube between $z^{\prime}=0$ and $z^{\prime}=z$. Applying the divergence theorem, we have

$$
\begin{aligned}
\int_{0}^{z} \int_{S\left(z^{\prime}\right)}\left(\tau_{z z}+\nabla^{2} \tau\right) d S d z^{\prime} & =\left.\int_{S\left(z^{\prime}\right)} \tilde{\nabla} \tau \cdot \mathbf{n} d S\right|_{0} ^{z} \\
& =\left.\int_{S\left(z^{\prime}\right)} \tau_{z} d S\right|_{0} ^{z} \\
& =\left.\int_{S\left(z^{\prime}\right)} \frac{\cos \theta}{v} d S\right|_{0} ^{z},
\end{aligned}
$$

where $\theta$ is the angle between the ray and the $z$-axis.

Thus, letting $S$ be an infinitesimal element of area cut out by the tube of rays on the plane $z=$ constant, we have

$$
\begin{aligned}
\int_{S\left(z^{\prime}\right)}\left(\tau_{z z}+\nabla^{2} \tau\right) d S & =\left.\frac{d}{d z} \int_{S\left(z^{\prime}\right)} \frac{\cos \theta}{v} d S\right|_{0} ^{z}, \\
\tau_{z z}+\nabla^{2} \tau & =\frac{1}{S} \frac{d}{d z}\left(\frac{S t_{0}}{v}\right),
\end{aligned}
$$

where $t_{0}=\cos \theta$. Substituting equation A-9 into the transport equation $\mathrm{A}-7$, along a ray we have

$$
\begin{aligned}
\frac{d}{d z}\left(\log A^{2}\right)+\frac{v}{S t_{0}} \frac{d}{d z}\left(\frac{S t_{0}}{v}\right) & =0 \\
\frac{A^{2} S t_{0}}{v} & =\text { Constant }
\end{aligned}
$$

But $\operatorname{det}\{\boldsymbol{C}\}=S$, and so we have 
$A\left(z ; \boldsymbol{x}_{s}, \boldsymbol{t}_{s}\right)=\sqrt{\frac{v\left[z, \boldsymbol{X}\left(z ; \boldsymbol{x}_{s}, \boldsymbol{t}_{s}\right)\right] t_{s, 0} \operatorname{det}\left\{\boldsymbol{C}\left(0 ; \boldsymbol{x}_{s}, \boldsymbol{t}_{s}\right)\right\}}{v\left(0, \boldsymbol{x}_{s}\right) t_{0}\left(z ; \boldsymbol{x}_{s}, \boldsymbol{t}_{s}\right) \operatorname{det}\left\{\boldsymbol{C}\left(z ; \boldsymbol{x}_{s}, \boldsymbol{t}_{s}\right)\right\}}}$,

which is nonzero everywhere. Here, we have normalized $A(0)$ to 1. Recall that $t_{s, 0} \operatorname{det}\left\{\boldsymbol{C}\left(0 ; \boldsymbol{x}_{s}, \boldsymbol{t}_{s}\right)\right\}$ is the differential area of a right cross section of the tube of rays at the source. We shall assume it is independent of $\boldsymbol{x}_{\mathrm{s}}$ and of $\boldsymbol{v}_{\mathrm{s}}$.

\section{APPENDIX B}

\section{THE FACTOR $\Psi_{0}$}

We have the ray tracing system for the constant velocity case, i.e., $v(z, \boldsymbol{x})=v_{0}$,

$$
\begin{aligned}
& \frac{d \boldsymbol{X}}{d z}(z)=\dot{X}(z)=H_{\boldsymbol{p}}=\frac{\boldsymbol{p}}{\sqrt{\frac{1}{v_{0}^{2}}-\boldsymbol{p}^{2}}}=\frac{\boldsymbol{t}}{t_{0}}=\boldsymbol{v},\left.\quad \boldsymbol{X}\right|_{z=0}=\boldsymbol{x}_{s} ; \\
& \frac{d \boldsymbol{p}}{d z}(z)=\dot{\boldsymbol{p}}(z)=\frac{\dot{\boldsymbol{t}}(z)}{v_{0}}=\mathbf{0} ; \\
& \frac{d T}{d z}(z)=\dot{T}(z)=\frac{1}{v t_{0}}=\frac{\mu}{v},\left.\quad T\right|_{z=0}=\tau_{0}\left(\boldsymbol{x}_{s}\right) ;
\end{aligned}
$$

The dynamic ray tracing system is given by

$$
\left.\frac{d \boldsymbol{B}}{d z}=\mathbf{0}, \quad \frac{d \boldsymbol{C}}{d z}=v_{0} \mu\left[\boldsymbol{Q}_{\perp}+\mu^{2} \boldsymbol{Q}_{\|}\right)\right] \boldsymbol{B},
$$

with the initial conditions

$$
\left.\boldsymbol{B}\right|_{z=0}=\left.\mathrm{i} \epsilon\left(\boldsymbol{Q}_{\perp}+\frac{1}{\mu} \boldsymbol{Q}_{\|}\right) \quad \boldsymbol{C}\right|_{z=0}=\boldsymbol{Q}_{\perp}+\mu \boldsymbol{Q}_{\|} .
$$

Solving these two systems, we have

$$
\begin{array}{ll}
\boldsymbol{x}(z)=\boldsymbol{x}_{s}+z \boldsymbol{v}, \quad \boldsymbol{t}=\boldsymbol{t}_{s}, & \tau(z)=\frac{z \mu}{v_{0}}=\frac{r}{v_{0}} \\
\boldsymbol{B}(z)=\mathrm{i} \epsilon\left(\boldsymbol{Q}_{\perp}+\frac{1}{\mu} \boldsymbol{Q}_{\|}\right), & \boldsymbol{C}(z)=a\left[\boldsymbol{Q}_{\perp}+\mu \boldsymbol{Q}_{\|}\right],
\end{array}
$$

where we have written

$$
r=z \mu, \quad \text { and } \quad a=1+\mathrm{i} \epsilon v_{0} r .
$$

We note that

$$
\boldsymbol{B}(z) \boldsymbol{C}(z)^{-1}=\frac{\mathrm{i} \epsilon}{a}\left(\boldsymbol{Q}_{\perp}+\frac{1}{\mu^{2}} \boldsymbol{Q}_{\|}\right) .
$$

For $\boldsymbol{x}$ near the point $\boldsymbol{x}_{s}+z \boldsymbol{v}$ on the central ray parameterized by $\boldsymbol{x}_{s}, \boldsymbol{t}_{s}$, we have

$$
\begin{aligned}
T\left(z, \boldsymbol{x} ; \boldsymbol{x}_{s}, \boldsymbol{t}_{s}\right)= & \frac{z \mu}{v_{0}}+\frac{1}{v_{0} \mu} \boldsymbol{\nu}^{T}\left(\boldsymbol{x}-\boldsymbol{x}_{s}-z \boldsymbol{v}\right)+\frac{\mathrm{i} \epsilon}{2 a}\left(\boldsymbol{x}-\boldsymbol{x}_{s}\right. \\
& -z \boldsymbol{v})^{T}\left(\boldsymbol{Q}_{\perp}+\frac{1}{\mu^{2}} \boldsymbol{Q}_{\|}\right)\left(\boldsymbol{x}-\boldsymbol{x}_{s}-z \boldsymbol{v}\right) .
\end{aligned}
$$

From equation 25 the wavefield resulting from the beam through $\left(\boldsymbol{x}_{s}, \boldsymbol{t}_{s}\right)$ is
$\Psi\left(z, \boldsymbol{x} ; \boldsymbol{x}_{s}, \boldsymbol{t}_{s}\right)=\frac{\Psi_{0}}{\sqrt{t_{0}(z) \operatorname{det}\{\boldsymbol{C}(z)\}}} \exp \left[\mathrm{i} \omega T\left(z, \boldsymbol{x} ; \boldsymbol{x}_{s}, \boldsymbol{t}_{s}\right)\right]$,

where the velocities have canceled because $v=v_{0}=$ constant and

$$
\sqrt{t_{0} \operatorname{det}\{\boldsymbol{C}\}}=a^{\bar{n} / 2} \text {. }
$$

Integrating all the beams for $\tilde{\boldsymbol{t}}=\left(t_{0}, \boldsymbol{t}\right)$ on the hemisphere $t_{0}>0$ using $\nu_{1}, \ldots, \nu_{\bar{n}}$ as variables of integration, we have

$$
\Psi\left(z, \boldsymbol{x} ; \boldsymbol{x}_{s}\right)=\int_{-\infty}^{\infty} \ldots \int_{-\infty}^{\infty} \Psi\left(z, \boldsymbol{x} ; \boldsymbol{x}_{s}, \boldsymbol{v}\right) \frac{\mathrm{d} \nu_{1} \ldots \mathrm{d} \nu_{\bar{n}}}{\mu^{n}} .
$$

Here,

$$
\frac{\mathrm{d} \nu_{1} \ldots \mathrm{d} \nu_{\bar{n}}}{\mu^{n}}
$$

is the differential element of solid angle. The integrand is seen from equation B-9 to have the form

$$
\Psi\left(z, x ; x_{s}, \boldsymbol{v}\right) \equiv g\left(z, \boldsymbol{x} ; \boldsymbol{x}_{s}, \boldsymbol{v}\right) \exp \left(i \omega T\left(z, \boldsymbol{x} ; \boldsymbol{x}_{s}, \boldsymbol{v}\right)\right),
$$

where

$$
g\left(z, \boldsymbol{x} ; \boldsymbol{x}_{s}, \boldsymbol{v}\right)=\frac{\Psi_{0}}{a^{\bar{n} / 2} \mu^{n}} .
$$

To apply the multidimensional saddle point method, we need the following quantities evaluated at the stationary point $\boldsymbol{v}=\boldsymbol{x} / z$ :

$$
\begin{aligned}
& g_{0}=\left.g\left(z, \boldsymbol{x} ; \boldsymbol{x}_{s}, \boldsymbol{v}\right)\right|_{\boldsymbol{\nu}=\left(\boldsymbol{x}-\boldsymbol{x}_{s}\right) / z}=\frac{\Psi_{0}}{a^{\bar{n} / 2} \mu^{n}}, \\
& T_{0}=\left.T\left(z, \boldsymbol{x} ; \boldsymbol{x}_{s}, \boldsymbol{v}\right)\right|_{\boldsymbol{\nu}=\left(\boldsymbol{x}-\boldsymbol{x}_{s}\right) / z}=\frac{r}{v_{0}}, \\
& T_{0, \boldsymbol{v}}=\left.T_{\boldsymbol{v}}\left(z, \boldsymbol{x} ; \boldsymbol{x}_{s}, \boldsymbol{v}\right)\right|_{\boldsymbol{\nu}=\left(\boldsymbol{x}-\boldsymbol{x}_{s}\right) / z}=\mathbf{0}, \\
& T_{0, \boldsymbol{\nu} \boldsymbol{v}}=\left.T_{\boldsymbol{\nu} \boldsymbol{v}}\left(z, \boldsymbol{x} ; \boldsymbol{x}_{s}, \boldsymbol{v}\right)\right|_{\boldsymbol{\nu}=\left(\boldsymbol{x}-\boldsymbol{x}_{s}\right) / z}=-\frac{r}{v_{0} a \mu^{2}} \boldsymbol{Q}_{\perp} \\
&-\frac{r}{v_{0} \mu^{4} a} \boldsymbol{Q}_{\|}, \\
& \text {so that } \operatorname{det}\left\{T_{0, \boldsymbol{\nu} \boldsymbol{v}}\right\}=\left(\frac{-r}{v_{0} a}\right)^{\bar{n}} \frac{1}{\mu^{2 n}},
\end{aligned}
$$

as we see from equation B-8 on differentiating with respect to $\nu_{1}, \ldots, \nu_{\bar{n}}$ holding $z, \boldsymbol{x}, \boldsymbol{x}_{s}$ fixed. Thus, the stationary point is $\boldsymbol{v}=(\boldsymbol{x}$ $\left.-\boldsymbol{x}_{s}\right) / z$, and by the stationary phase method, we have asymptotically for large $\omega$

$$
\begin{aligned}
\Psi(x, z) & \approx\left(\frac{2 \pi}{\omega}\right)^{\bar{n} / 2} \frac{g_{0}}{\sqrt{\operatorname{det}\left\{T_{0, \boldsymbol{\nu} v}\right\}}} \exp \left(\mathrm{i} \omega T_{0}\right) \exp \left(-\frac{\mathrm{i} \bar{n} \pi}{4}\right) \\
& =\Psi_{0}\left(\frac{2 \pi v_{0}}{\omega r}\right)^{n-1 / 2} \exp \left[\mathrm{i}\left(\frac{\omega r}{v_{0}}-\frac{(n-1) \pi}{4}\right)\right] .
\end{aligned}
$$

We require that $\Psi(x, z)$ match with the large argument asymptotics of the Green's function (see Appendix C) 


$$
\begin{aligned}
& \frac{\mathrm{i}}{4}\left(\frac{\omega}{2 \pi v_{0} r}\right)^{n / 2-1} H_{n / 2-1}^{(1)}\left(\frac{\omega r}{v_{0}}\right) \\
& \quad \sim \frac{\mathrm{i}}{4}\left(\frac{\omega}{2 v_{0}}\right)^{n-3 / 2}\left(\frac{1}{\pi r}\right)^{n-1 / 2} \exp \left[\mathrm{i}\left(\frac{\omega r}{v_{0}}-\frac{(n-1) \pi}{4}\right)\right]
\end{aligned}
$$

Hence, we get

$$
\Psi_{0}=\frac{\mathrm{i}}{4 \pi}\left(\frac{\omega}{2 \pi v_{0}}\right)^{n-2}
$$

Thus, we have

$$
\Psi_{0}=\left\{\begin{array}{ll}
\frac{\mathrm{i}}{4 \pi} & \text { for } n=2 \\
\frac{\mathrm{i} \omega}{8 \pi^{2} v_{0}} & \text { for } n=3
\end{array} .\right.
$$

Thus, from equations 25 and B-18 we have $\Psi$ :

$$
\begin{aligned}
& \Psi\left(z, \boldsymbol{x} ; \boldsymbol{x}_{s}, \boldsymbol{v}_{s}\right) \\
& \approx \frac{\mathrm{i}}{4 \pi}\left(\frac{\omega}{2 \pi v\left(0, \boldsymbol{x}_{s}\right)}\right)^{n-2} \sqrt{\frac{v(z, \boldsymbol{x})}{v\left(0, \boldsymbol{x}_{s}\right) t_{0}(z) \operatorname{det}\{\boldsymbol{C}(z)\}}} \\
& \times \exp \left[\mathrm{i} \omega T\left(z, \boldsymbol{x} ; \boldsymbol{x}_{s}, \boldsymbol{t}_{s}\right)\right] .
\end{aligned}
$$

\section{APPENDIX C}

\section{THE CONSTANT $\Psi_{0}$ AND THE CONSTANT VELOCITY GREEN'S FUNCTION}

It is easily confirmed that

$$
G_{n}(r)=\frac{\mathrm{i}}{4}\left(\frac{\omega}{2 \pi v_{0} r}\right)^{n / 2-1} H_{n / 2-1}^{(1)}\left(\frac{\omega r}{v_{0}}\right)
$$

satisfies the Helmholtz equation,

$$
\tilde{\nabla}^{2} G_{n}+\frac{\omega^{2}}{v_{0}^{2}} G_{n}=0
$$

except possibly at the origin. We shall verify that

$$
\widetilde{\boldsymbol{\nabla}}^{2} G_{n}+\frac{\omega^{2}}{v_{0}^{2}} G_{n}=-\boldsymbol{\delta}(\widetilde{\boldsymbol{x}}),
$$

where $\widetilde{\boldsymbol{\delta}}(\widetilde{\boldsymbol{x}})$ is the $n$-dimensional Dirac delta function

$$
\widetilde{\boldsymbol{\delta}}(\widetilde{\boldsymbol{x}})=\delta(z) \delta\left(x_{1}\right) \ldots \delta\left(x_{\bar{n}}\right) .
$$

We first consider the integral of $\widetilde{\nabla}^{2} G_{n}+\omega^{2} / v_{0}^{2} G_{n}$ over the interior $V_{r}$ of a small sphere $S_{r}$ of radius $\mathrm{r}$ and centered at the origin. By applying the divergence theorem to the first term, we obtain

$$
\int_{V_{r}} \tilde{\nabla}^{2} G_{n}+\frac{\omega^{2}}{v_{0}^{2}} G_{n} \mathrm{~d} V=\int_{S_{r}} \frac{\mathrm{d} G_{n}}{\mathrm{~d} r} \mathrm{~d} A+\int_{V_{r}} \frac{\omega^{2}}{v_{0}^{2}} G_{n} \mathrm{~d} V,
$$

where $V_{r}, S_{r}, \mathrm{~d} V$, and $\mathrm{d} A$ are respectively the $n$-dimensional volume interior to the sphere $S_{r}$, its $\bar{n}$-dimensional surface, the differential volume element in $V_{r}$, and the differential area element on $S_{r}$. We shall let $r$ tend to zero, but first we need to study the asymptotic form of $G_{N}(r)$ as $r \rightarrow 0$. We find from Abramowitz and Stegun (1965), formulas 9.1 .8 and 9.1.9, that

$$
\begin{gathered}
H_{0}^{(1)}\left(\frac{\omega r}{v_{0}}\right) \sim \frac{2 \mathrm{i}}{\pi} \log (r), \\
H_{n / 2-1}^{(1)}\left(\frac{\omega r}{v_{0}}\right) \sim-\frac{\mathrm{i}}{\pi} \Gamma\left(\frac{n}{2}-1\right)\left(\frac{2 v_{0}}{\omega r}\right)^{n / 2-1}
\end{gathered}
$$

Thus,

$$
G_{2}(r) \sim-\frac{1}{2 \pi} \log (r), \quad G_{n}(r) \sim \frac{\Gamma\left(\frac{n}{2}-1\right)}{4 \pi} \frac{1}{\pi^{n / 2-1} r^{n-2}},
$$

with derivative

$$
\frac{\mathrm{d} G_{n}}{\mathrm{~d} r} \sim-\frac{\left(\frac{n}{2}-1\right) !}{2 \pi} \frac{1}{\pi^{n / 2-1} r^{n-1}}
$$

as $r \rightarrow 0$ and is true also for $n=2$. But the surface area of $S_{r}$ is $2 \pi^{n / 2} r^{n-1} /(n / 2-1)$ ! and so as $r \rightarrow 0$ the first integral on the right of equation $\mathrm{C}-5$ tends to -1 , and the second integral tends to zero. This verifies equation $\mathrm{C}-3$.

Let us now examine the large argument asymptotic approximation to $G_{n}(r)$. Using Abramowitz and Stegun (1965) formula 9.2.3

$$
H_{n / 2-1}^{(1)}\left(\frac{\omega r}{v_{0}}\right) \sim \sqrt{\frac{2 v_{0}}{\pi \omega r}} \exp \left[\mathrm{i}\left(\frac{\omega r}{v_{0}}-\frac{(n-1) \pi}{4}\right)\right],
$$

we find that as $r \rightarrow \infty$

$$
\begin{aligned}
G_{n}(r) \sim & -\frac{1}{4}\left(\frac{\omega}{2 v_{0}}\right)^{n-3 / 2}\left(\frac{1}{\pi r}\right)^{n-1 / 2} \\
& \times \exp \left[\mathrm{i}\left(\frac{\omega r}{v_{0}}-\frac{(n+1) \pi}{4}\right)\right] .
\end{aligned}
$$

\section{APPENDIX D}

\section{THE STORMER/VERLET SCHEME}

Consider the following Hamiltonian system

$$
\frac{d p}{d t}=-\nabla_{q} H(p, q), \quad \frac{d q}{d t}=\nabla_{p} H(p, q),
$$

where $H(p, q)$ is a smooth function defined on an open set $\Omega \subset \mathbb{R}^{d \times d}$, with the initial conditions $p(t=0)=p_{0}$ and $q(t=0)=q_{0}$. To achieve a higher order of accuracy, we consider the following Stormer/Verlet scheme (Hairer et al., 2002):

$$
q^{n+1 / 2}=q^{n}+\frac{\Delta t}{2} \nabla_{p} H\left(p^{n}, q^{n+1 / 2}\right)
$$




$$
p^{n+1}=p^{n}-\frac{\Delta t}{2}\left(\nabla_{q} H\left(p^{n}, q^{n+1 / 2}\right)+\nabla_{p} H\left(p^{n+1}, q^{n+1 / 2}\right)\right)
$$

$$
q^{n+1}=q^{n+1 / 2}+\frac{\Delta t}{2} \nabla_{p} H\left(p^{n+1}, q^{n+1 / 2}\right) .
$$

Here, equation D-4 is explicit, but equation D-2 and D-3 are implicit in both $p^{n+1}$ and $q^{n+1 / 2}$. Numerically, we solve them using Newton's method with a forward Euler step as the initial guess $\left(\widetilde{p}^{n+1}, \widetilde{q}^{n+1 / 2}\right)$, i.e.,

$\tilde{p}^{n+1}=p^{n}-\Delta t \nabla_{q} H\left(p^{n}, q^{n}\right), \quad \widetilde{q}^{n+1 / 2}=q^{n}+\frac{\Delta t}{2} \nabla_{p} H\left(p^{n}, q^{n}\right)$.

This numerical method is second-order accurate, very simple to implement, and also preserves the symplectic structure.

\section{REFERENCES}

Abramowitz, M., and I. A. Stegun, 1965, Handbook of mathematical functions: Dover Publications, Inc..

Albertin, U., D. Yingst, P. Kitchenside, and V. Tcheverda, 2004, True-amplitude beam migration: 74th Annual International meeting SEG, Expanded Abstracts, 949-952.

Alkhalifah, T., 1995, Gaussian beam depth migration for anisotropic media: Geophysics, 60, 1474-1484.

Cerveny, V., M. Popov, and I. Psencik, 1982, Computation of wave fields in inhomogeneous media-Gaussian beam approach: Geophysical Journal of the Royal Astronomical Society, 70, 109-128.

George, T., J. Virieux, and R. Madariaga, 1987, Seismic wave synthesis by Gaussian beam summation: A comparison with finite differences, Geophysics, 52, 1065-1073.

Gray, S., 2005, Gaussian beam migration of common shot records: Geophysics, 70, S71-S77.

Gray, S., and W. May, 1994, Kirchhoff migration using eikonal equation traveltimes: Geophysics, 59, 810-817.

Hairer, E., C. Lubich, and G. Wanner, 2002, Geometric numerical integration: Structure-preserving algorithms for ordinary differential equations: Springer-Verlag.

Hale, D., 1992, Migration by the Kirchhoff, slant stack, and Gaussian beam methods: Colorado School of Mines, Center for Wave Phenomena, Report 121.

Han, Q., and R. S. Wu, 2005, Common image gathers in the plane-wave domain: A prestack Gaussian beam migration algorithm: 75th Annul International Meeting, SEG, Expanded Abstracts, 2021-2024.

Hill, N., 1990, Gaussian beam migration: Geophysics, 55, 1416-1428.

, 2001, Prestack gaussian-beam depth migration: Geophysics, 66, $1240-1250$.

Hormander, L., 1971, On the existence and the regularity of solutions of linear pseudo-differential equations: L'Enseignement Mathematique, 17, 99-163.

Leung, S., J. Qian, and S. Osher, 2004, A level set method for three dimensional paraxial geometrical optics with multiple sources: Communication in Mathematical Sciences, 2, 657-686.

Norris, A., B. White, and J. Schrieffer, 1987, Gaussian wave packets in inhomogeneous media with curved interfaces: Proceedings of the Royal Society of London, Series A, Mathematical and Physical Sciences, 412, 93-123.

Protasov, M., and V. Tcheverda, 2006, True/preserving amplitude seismic imaging based on Gaussian beam application: 76th Annual International Meeting, SEG, Expanded Abstracts, 2126-2129.

Qian, J., and S. Leung, 2004, A level set based Eulerian method for paraxial multivalued traveltimes: Journal of Computational Physics, 197, 711-736.

, 2006, A local level set method for paraxial multivalued geometric optics: SIAM Journal of Scientific Computing, 28, 206-223.

Qian, J., and W. W. Symes, 2002, Adaptive finite difference method for traveltime and amplitude: Geophysics, 67, 167-176.

Ralston, J., 1983, Gaussian beams and the propagation of singularities, in W. Littman, ed., Studies in partial differential equations: Mathematics Association of America Studies in Mathematics, 23, 206-248.

Symes, W. W., and J. Qian, 2003, A slowness matching Eulerian method for multivalued solutions of eikonal equations: Journal of Scientific Computing, 19, 501-526.

Tanushev, N., J. Qian, and J. Ralston, 2007, Mountain waves and Gaussian beams: SIAM Journal of Multiscale Modeling and Simulation (to appear).

White, B., A. Norris, A. Bayliss, and R. Burridge, 1987, Some remarks on the Gaussian beam summation method: Geophysical Journal of the Royal Astronomical Society, 89, 579-636.

Zacek, K., 2005, Gaussian packet prestack depth migration of the Marmousi data set: 75th Annual International Meeting, SEG, Expanded Abstracts, $1822-1825$ 